\title{
SPATIAL DISTRIBUTION OF STARS AROUND SIX METAL-POOR GLOBULAR CLUSTERS IN THE GALACTIC BULGE
}

\author{
Cho-Rhong Chang ${ }^{1}$, Jae-Woo Kim ${ }^{2}$, Noriyuki Matsunaga ${ }^{3}$, Minwa Han $^{1}$, Jongwan Ko ${ }^{4}$, Sang-Hyun Chun $^{5}$, \\ MinheE $\mathrm{KANG}^{1}$, AND YOUnG-Jong SOHN ${ }^{1}$ \\ 1 Department of Astronomy, Yonsei University, Seoul 120-749, Korea \\ E-mail : sohnyj@yonsei.ac.kr \\ 2 Center for the Exploration of the Origin of the Universe (CEOU), Department of Physics and Astronomy, \\ Seoul National University, Seoul 151-742, Korea \\ ${ }^{3}$ Kiso Observatory, Institute of Astronomy, the University of Tokyo, 10762-30 Mitake, Kiso, \\ Nagano 397-0101, Japan \\ ${ }^{4}$ Korea Astronomy and Space Science Institute, Daejeon 305-348, Korea \\ ${ }^{5}$ Yonsei University Observatory, Seoul 120-749, Korea \\ (Received September 18, 2013; Accepted October 30, 2013)
}

\begin{abstract}
Wide-field $J H K_{s}$ images obtained with the SIRIUS near-infrared camera of the IRSF $1.4 \mathrm{~m}$ telescope are used to examine the tidal structures of the spatial stellar configuration around six metal-poor $([\mathrm{Fe} / \mathrm{H}]<-1.0)$ globular clusters located within $3 \mathrm{kpc}$ from the Galactic center. The radial surface density profiles are obtained from the surface photometry of the cluster images and the star counting for the photometric data. For the star counting, candidates of cluster member stars are selected with an filtering algorithm in color-magnitude diagrams. We find that the six target clusters show tidal overdensity features in the radial surface density profiles. There is a break inside the tidal radius for each cluster, and the profile in the outer overdensity region is characterized by a power law. Twodimensional density maps of all the clusters show distorted asymmetric stellar configurations in the outer region. In five out of the six target clusters, the overdensity features are likely to be associated with the effects of the Galaxy dynamical interaction and the cluster space motions. The observed tidal configurations of stars suggest that several metal-poor clusters in the Galactic bulge are possibly surviving remnants of mergers to build the old stellar system of the Galactic bulge.
\end{abstract}

Key words : Galaxy: bulge — globular clusters: general — globular cluster

\section{INTRODUCTION}

According to the hierarchical clustering scenario, the galaxy formation is thought to result from repeated merging events and disruptions of many smaller satellite fragments. In this paradigm, it is commonly accepted that the some globular clusters in the Galaxy are survivors of initially more numerous dwarf satellite galaxies, depopulated by tidal disruption processes (Murali \& Weinberg 1997a,b; Fall \& Zhang 2001). Indeed, the spatial alignment of the globular cluster system through the Galactic halo is the observational evidence for the gradual merging events of dwarf satellites. Lynden-Bell (1982) suggested that several dwarf spheroidal satellites of the Galaxy are placed on two great streams, i.e., the Magellanic stream and the Fornax-Leo-Sculptor stream, and interpreted this phenomenon as a result of the accretion of external fragments. Furthermore, apparent stellar substructural streams in the Galactic halo in the form of both globular clusters and a population of dwarf galaxies have

Corresponding Author: S.-H. Chun been mapped by several large scale surveys of the stellar substructure of the Galactic halo, such as the Two Micron All Sky Survey (2MASS), the Sloan Digital Sky Survey (SDSS), and the Sloan Extension for Galactic Understanding and Exploration (SEGUE) (Ivezić et al. 2000; Yanny et al. 2000, 2003; Newberg et al. 2002, 2009, 2010; Martin et al. 2004; Rocha-Pinto et al. 2004; Belokurov et al. 2006b, 2007a,b; Grillmair 2006, 2009; Grillmair \& Dionatos 2006; Grillmair \& Johnson 2006; Jurić et al. 2008). The most striking example is the discovery of the trails of stellar debris and globular clusters associated with the Sagittarius dwarf galaxy (Ibata et al. 1994, 1995, 1997, 2001; Majewski et al. 2003; Newberg et al. 2003; Belokurov et al. 2006b; Yanny et al. 2009). Recently, Law \& Majewski (2010) suggested that the initial Sagittarius system may have contained five to nine globular clusters in their numerical models of the tidal disruption of the Sagittarius dwarf galaxy.

Discoveries of multiple stellar populations in globular clusters have also suggested that several globular clusters are possible relics of the hierarchical assembly of the Galactic halo and bulge. The most vivid sample is $\omega$-Centauri, in which distinct components in 
main-sequence, sub-giant branch, and red-giant branch (RGB) stars are characterized by different metallicities and ages (e.g., Lee et al. 1999; Pancino et al. 2000; Bedin et al. 2004; Sollima et al. 2005; Villanova et al. 2007). Other samples include NGC 1851 (Milone et al. 2008; Han et al. 2009; Lee et al. 2009b; Ventura et al. 2009), NGC 2808 (D'Antona et al. 2005; Lee et al. 2005; Piotto et al. 2007), NGC 6388 (Piotto 2008; Moretti et al. 2009), and NGC 6656 (Marino et al. 2009). Recently, Lee et al. (2009a) measured the Ca abundance of stars in globular clusters using Ca uvby photometry, and reported that seven clusters (i.e., $\omega$-Centauri, NGC 6656, NGC 1851, NGC 2808, M4, M5, NGC 6752) show discrete RGB sequences with a large spread in Ca abundance. They also noted that all the globular clusters with signs of multiple stellar populations have a relatively extended horizontal branch (EHB) in their colormagnitude diagrams (CMDs) (Lee et al. 2007). While most of the globular clusters with multiple populations have been identified at high Galactic latitudes, Ferraro et al. (2009) found two different stellar populations in a globular-cluster-like system Terzan 5 in the Galactic bulge, and suggested that other similar objects might be hidden in the central region of the Galaxy.

Extended tidal features in a spatial stellar density distribution around globular clusters, such as a tidal tail structure, might also play a key role in disentangling the possibility that the stellar Galactic halo and bulge may have formed from the accretion of dwarf satellites. If Galactic globular clusters formed within larger systems, they are likely to be surrounded by external halos and tails made up of stars that were tidally stripped from their parent systems through internal and external dynamical processes (e.g., Gnedin \& Ostriker 1997; Murali \& Weinberg 1997b). Indeed, numerical simulations have indicated that the clustergalaxy interaction can cause stars to escape from the cluster gravitational potential. The unbounded stars show a tendency to remain in the vicinity of the cluster for several orbital periods and become tidal debrisforming tails (Combes et al. 1999; Yim \& Lee 2002; Capuzzo Dolcetta et al. 2005; Montuori et al. 2007). The tidal tails are also found to be aligned with the cluster orbit, because the debris produced by a dissolving globular cluster will have similar orbital characteristics as the cluster itself. The extended tidal tails around globular clusters were first observed in wide-field photographic surveys (Grillmair et al. 1995; Leon et al. 2000), and now more than 30 of globular clusters in the Galaxy and M31 show extended tidal tails around them (Grillmair et al. 1996; Lehmann \& Scholz 1997; Testa et al. 2000; Siegel et al. 2001; Lee et al. 2003; Sohn et al. 2003; Lauchner et al. 2006; Chun et al. 2010, 2012; Jordi \& Grebel 2010). Recent wide-field CCD photometric surveys have revealed tidal structures stretching many degrees beyond the cluster tidal radius. The most spectacular examples are Palomar 5 (Odenkirchen et al. 2001, 2003, 2009; Rockosi et al. 2002; Grillmair \& Dionatos 2006) and NGC 5466 (Belokurov et al. 2006a;

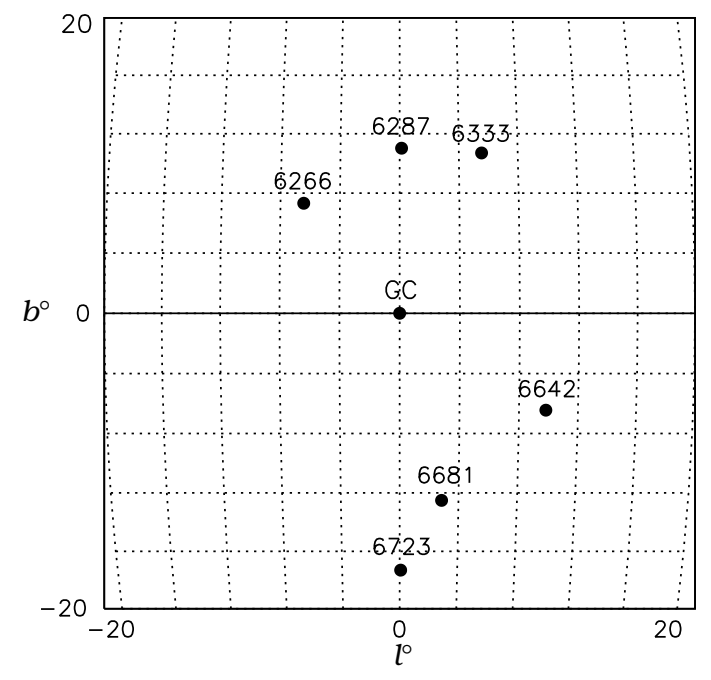

Fig. 1.- Positions of the observed six target clusters in the Galactic coordinates. The straight line indicates the Galactic plane, and the Galactic center is represented as GC.

Grillmair \& Johnson 2006), which have tails that extend as much as $22^{\circ}$ and $45^{\circ}$, respectively. More interestingly, Chun et al. (2010) found a tidal bridge-like feature and an envelope structure around the pair of globular clusters M53 and NGC 5053. In addition, distorted tidal extensions also appear in two-dimensional iso-density contour maps for stars in several clusters at locations even inside their tidal radii (e.g., Grillmair et al. 1996; Leon et al. 2000; Jordi \& Grebel 2010).

Most of these previous tidal structure studies focused on metal-poor globular clusters in the Galactic halo in order to understand the structure and formation of the stellar system in the Galactic halo. We note, however, that metal-poor globular clusters are equally present from the central bulge to the outer halo, although metal-rich globular clusters appear to be associated with the central bulge or inner spheroid components in the Galaxy (Minniti 1995; Côté 1999; Côté et al. 2000; Bica et al. 2006). Metal-poor globular clusters in the central region of the Galaxy can be important objects for understanding the early evolution of the Galaxy, including bulge formation. Thus, searching for any tidal overdensity structure around metalpoor globular clusters in the Galactic bulge plays a key role in understanding their origins, whether they were formed inside the Galaxy or accreted into the central region of the Galaxy through mergers of subclumps at early epochs (Baugh et al. 1996; Aguerri et al. 2001; Nakasato \& Nomoto 2003). Unfortunately, searching for a tidal structure around metal-poor globular clusters in the bulge region has been poorly studied because of the high value of extinction and field star contamination. Recently, Chun et al. (2012) attempted to 
Table 1.

Basic information for the six target clusters

\begin{tabular}{|c|c|c|c|c|c|c|c|c|c|}
\hline Target & $\begin{array}{c}\alpha(\mathrm{J} 2000) \\
(\mathrm{h} \mathrm{m}\end{array}$ & $\begin{array}{c}\delta(\mathrm{J} 2000) \\
\left(\begin{array}{cc}0^{\circ} & \prime \prime\end{array}\right)\end{array}$ & $\begin{array}{c}1 \\
(\mathrm{deg})\end{array}$ & $\begin{array}{c}\mathrm{b} \\
(\mathrm{deg})\end{array}$ & $\begin{array}{l}R_{G C} \\
(\mathrm{kpc})\end{array}$ & $\begin{array}{l}r_{c} \\
\left({ }^{\prime}\right)\end{array}$ & $\begin{array}{l}r_{t} \\
(')\end{array}$ & {$[\mathrm{Fe} / \mathrm{H}]$} & $\overline{E(B-V)}$ \\
\hline NGC 6266 & 170112.8 & -300649 & 353.57 & 7.32 & 1.7 & 0.18 & 8.97 & -1.29 & $0.35 \sim 0.51$ \\
\hline NGC & 170 & $-2242 \quad 29$ & 0.13 & 11.02 & 2.1 & 0.26 & 10.51 & -2.05 & .82 \\
\hline NGC 6333 & 171911.8 & -183059 & 5.54 & 10.70 & 1.7 & 0.5 & 8.16 & -1.75 & $0.34 \sim 0.72$ \\
\hline NG & 183154.1 & -232831 & 9.81 & -6.44 & 1.7 & 0.10 & 10.07 & -1 & $0.36 \sim 0.50$ \\
\hline NGC 6681 & 184312.7 & -321731 & 2.85 & -12 & 2.1 & 0.03 & 7.91 & -1 & $0.10 \sim 0.14$ \\
\hline NGC 6723 & 185933.2 & -363754 & 0.07 & -17.30 & 2.6 & 0.94 & 10.51 & -1.12 & $0.11 \sim 0.75$ \\
\hline
\end{tabular}

Notes. $R_{G C}$ indicates the distance from the Galactic center. $r_{c}$ and $r_{t}$ are the core radius and tidal radius of a cluster, respectively. Data are taken from Harris (1996). The range of $E(B-V)$ were derived from Schlegel et al. (1998).

find the stellar substructure around the globular cluster NGC 6626 in the bulge region, and first found a stellar substructure extending toward the direction of the Galactic plane for the clusters in the bulge region. In the present paper, we define the bulge clusters as the area within $3 \mathrm{kpc}$ from the Galactic center. In the Galaxy, about 150 globular clusters are listed in the database of Harris (1996), which was revised in 2003. Among a total of 43 Galactic bulge globular clusters, there are 22 metal-poor $([\mathrm{Fe} / \mathrm{H}]<-1.0)$ and 21 metalrich $([\mathrm{Fe} / \mathrm{H}]>-1.0)$ globular clusters. On this basis, we have focused on obtaining a wide-field and moderately deep homogeneous photometric data set for the metal-poor bulge globular clusters in the near-infrared regime. Indeed, the effects of high extinction toward the bulge can be reduced by observing in the nearinfrared wavelengths, as the extinction in the $K$ band is only $\sim 10$ percent of that in the $V$ band (Rieke \& Lebofsky 1985).

In this paper, we investigate the spatial density distribution of stars around 6 metal-poor bulge clusters (NGC 6266, NGC 6287, NGC 6333, NGC 6642, NGC 6681, NGC 6723), and introduce the tidal structure in the vicinity of the clusters from the two-dimensional distribution of stars. Wide-field $\left(\sim 21^{\prime} \times 21^{\prime}\right) J H K_{s}$ photometric data for the target clusters have been secured using the 9-field mosaic imaging with the InfraRed Survey Facility (IRSF) $1.4 \mathrm{~m}$ telescope and the Simultaneous three-color InfraRed Imager for Unbiased Survey (SIRIUS) near-infrared camera. The target clusters with tidal radii in the range of $8^{\prime} \sim 10^{\prime}$ were selected in order to detect the tidal structure around the cluster tidal limits on the observed mosaic images. Table 1 provides the basic information of the target clusters. Fig. 1 indicates the positions of the target clusters in the Galactic coordinates. In Section 2, we present observations and data reduction procedures. The method for member star selection is described in Section 3. In Section 4, we investigate tidal features around the target clusters by using radial surface density profiles and two-dimensional stellar density maps.
Results are discussed in Section 5, and conclusions are summarized in Section 6.

\section{OBSERVATIONS AND DATA REDUC- TION}

Observations were carried out in July 2006, August 2007, and August 2009 using the SIRIUS nearIR camera mounted on the IRSF $1.4 \mathrm{~m}$ telescope at Sutherland, the South African Astronomical Observatory (SAAO). The SIRIUS camera contains three $1024 \times 1024 \mathrm{HgCdTe}(\mathrm{HAWAII})$ infrared arrays, which can take $J\left(\lambda_{c}=1.25 \mu \mathrm{m}\right), H\left(\lambda_{c}=1.63 \mu \mathrm{m}\right)$, and $K_{s}\left(\lambda_{c}=2.14 \mu \mathrm{m}\right)$ images simultaneously. The fieldof-view is $\sim 7^{\prime} .7 \times 7^{\prime} .7$ with a scale of $0^{\prime \prime} .45 \mathrm{pixel}^{-1}$. Further details about the camera can be found in Nagashima et al. (1999) and Nagayama et al. (2003).

We observed nine fields $(3 \times 3)$ for each target cluster, covering a total field-of-view of $\sim 21^{\prime} \times 21^{\prime}$. The cluster was centered on the central field and the other adjacent fields in the mosaic overlapped by $\sim 1^{\prime}$. In order to estimate the field star contamination (see Section 3 ), we also observed two comparison fields in the east- and west- regions over the cluster tidal radius, typically $15^{\prime} \sim 20^{\prime}$ away from the cluster center. Fig. 2 shows the pattern of the observation for a target cluster. For each position, three sets of ten dithered frames were obtained, for which each exposure has an integration time of 30 seconds. We also executed a set of ten dithered observations with an exposure time of 1.6 seconds for brighter sources. Dark frames and twilight flats were recorded at the beginning and the end of each run. Blank sky images at $(\alpha, \delta)=\left(17^{h} 11^{m} 24.7^{s},-27^{\circ} 27^{\prime} 20^{\prime \prime}\right.$ ; J2000.0) in the Pipe Nebula were also obtained to subtract the background radiation pattern of the sky. A summary of the observations is presented in Table 2 .

Preprocessing of raw data was performed using the SIRIUS pipeline (Nakajima, private communication), which is based on the NOAO Image Reduction and 
Table 2.

Observational log

\begin{tabular}{|c|c|c|c|c|c|}
\hline $\begin{array}{l}\text { Date } \\
\text { (Year) }\end{array}$ & Target & $\overline{\text { Field }}$ & 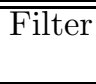 & $\begin{array}{c}\text { Exp. time } \\
(\mathrm{s})\end{array}$ & $\begin{array}{c}\text { FWHM } \\
\left({ }^{\prime \prime}\right)\end{array}$ \\
\hline \multirow[t]{6}{*}{2006} & NGC 6333 & $21^{\prime} \times 21^{\prime}$ & $J$ & $10 \times 1.6,3 \times 10 \times 30$ & $1.25,1.30$ \\
\hline & & & $H$ & $10 \times 1.6,3 \times 10 \times 30$ & $1.07,1.26$ \\
\hline & & & $K_{s}$ & $10 \times 1.6,3 \times 10 \times 30$ & $1.01,1.17$ \\
\hline & NGC 6723 & $21^{\prime} \times 21^{\prime}$ & $J$ & $10 \times 1.6,3 \times 10 \times 30$ & $1.26,1.13$ \\
\hline & & & $H$ & $10 \times 1.6,3 \times 10 \times 30$ & $1.02,0.98$ \\
\hline & & & $K_{s}$ & $10 \times 1.6,3 \times 10 \times 30$ & $0.97,0.94$ \\
\hline \multirow[t]{6}{*}{2007} & NGC 6287 & $21^{\prime} \times 21^{\prime}$ & $J$ & $10 \times 1.6,3 \times 10 \times 30$ & $1.70,1.53$ \\
\hline & & & $H$ & $10 \times 1.6,3 \times 10 \times 30$ & $1.50,1.40$ \\
\hline & & & $K_{s}$ & $10 \times 1.6,3 \times 10 \times 30$ & $1.41,1.32$ \\
\hline & NGC 6681 & $21^{\prime} \times 21^{\prime}$ & $J$ & $10 \times 1.6,3 \times 10 \times 30$ & $1.53,1.54$ \\
\hline & & & $H$ & $10 \times 1.6,3 \times 10 \times 30$ & $1.35,1.40$ \\
\hline & & & $K_{s}$ & $10 \times 1.6,3 \times 10 \times 30$ & $1.27,1.31$ \\
\hline \multirow[t]{6}{*}{2009} & NGC 6266 & $21^{\prime} \times 21^{\prime}$ & $J$ & $10 \times 1.6,3 \times 10 \times 30$ & $1.12,1.22$ \\
\hline & & & $H$ & $10 \times 1.6,3 \times 10 \times 30$ & $0.93,1.11$ \\
\hline & & & $K_{s}$ & $10 \times 1.6,3 \times 10 \times 30$ & $0.87,1.04$ \\
\hline & NGC 6642 & $21^{\prime} \times 21^{\prime}$ & $J$ & $10 \times 1.6,3 \times 10 \times 30$ & $1.57,1.21$ \\
\hline & & & $H$ & $10 \times 1.6,3 \times 10 \times 30$ & $1.46,1.12$ \\
\hline & & & $K_{s}$ & $10 \times 1.6,3 \times 10 \times 30$ & $1.36,1.05$ \\
\hline
\end{tabular}

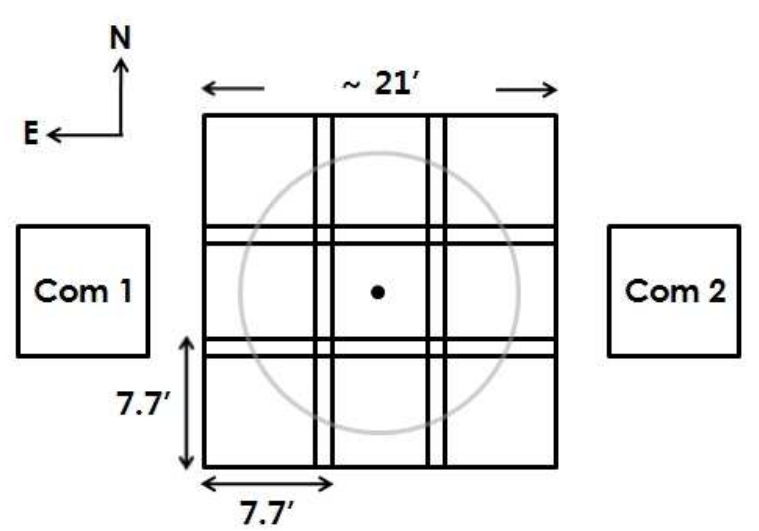

Fig. 2.- Layout of the 9 observed mosaic fields with a total field-of-view of $\sim 21^{\prime} \times 21^{\prime}$ and two comparison fields. The cluster is located at the center and the gray circle indicates the tidal radius of the cluster.

Analysis Facility (IRAF)* software package. First, a combined dark image was subtracted from every raw image, and then these dark-subtracted images were divided by a normalized twilight-flat image for each filter. From each flat-fielded image, we subtracted a sky frame obtained by a median combination of images for

*IRAF is distributed by the National Optical Astronomy Observatory, which is operated by the Association of Universities for Research in Astronomy, Inc., under a cooperative agreement with the USA National Science Foundation. the blank sky field. The next step was to combine the dithered images to obtain a higher signal-to-noise image for each position and each band. Finally, the mean background level, estimated by the mode of pixel intensity distribution, was subtracted from each combined image. The average seeing conditions of the reduced images at $J, H$, and $K_{s}$ were $1^{\prime \prime} .36,1^{\prime \prime} .22$, and $1^{\prime \prime} .14$ in FWHM, respectively.

The photometric measurements of stars were obtained with the point-spread-function (PSF) fitting programs DAOPHOT II and ALLSTAR (Stetson 1987; Stetson \& Harris 1988). A PSF which varies quadratically through positions was constructed for each frame using 20-30 bright, isolated, and unsaturated stars. The quality of each PSF was improved by removing neighboring stars around selected PSF stars and iteratively reconstructing the PSF. After the photometric measurements, DAOMATCH and DAOMASTER (Stetson 1992) were used to match up the coordinates of the stars in each frame with different filters and exposure times. The positional data for stars in the observed SIRIUS field were transformed into the equatorial coordinate system with reference to the positions of the point sources in the 2MASS catalog (Skrutskie et al. 2006). The astrometrical precision is better than $0^{\prime \prime} .22$ in most cases. In order to standardize the measured magnitudes, we compared the photometric results with those in the 2MASS catalog. The color term was ignored and a constant was added to fit our instrumental magnitudes into those in the 2MASS catalog. We note that the magnitudes we obtained are on the IRSF/SIRIUS natural system with the zero point cal- 
ibrated based on the 2MASS magnitudes. We then combined the data of the nine fields to construct the whole set of data for a target cluster. For stars in the overlapping regions of adjacent fields, photometric results with a small magnitude error in $K_{s}$ band were selected. Finally, an interstellar extinction correction for each star was executed using the Infrared Astronomy Satellite (IRAS) dust maps provided by Schlegel et al. (1998) and extinction coefficients from McCall (2004). We extracted reddening values $E(B-V)$ of individual stars from the dust maps and obtained extinctions for each filter using the reddening law. Ranges of $E(B-V)$ for each target cluster are listed in column 10 of Table 1 (see the two-dimensional dust extinction maps, Fig. 17 in Section 5). We then corrected the Galactic foreground extinctions by subtracting these values from the observed magnitudes. In this study, we denote the corrected magnitudes simply by $J, H$, and $K_{s}$.

A total of 113197, 36543, 57451, 122450, 34471 and 30629 stars were detected in each $\sim 21^{\prime} \times 21^{\prime}$ field of the target clusters NGC 6266, NGC 6287, NGC 6333, NGC 6642, NGC 6681, and NGC 6723, respectively. Fig. 3 shows the $(J-H, J)$ CMDs of stars observed in the vicinity of the target clusters. It is apparent that the CMDs for each cluster are severely contaminated by large number of stars belonging to the bulge and disk populations. As pointed out by Zoccali et al. (2003) and Vieira et al. (2007), the bulge horizontal branch $(\mathrm{HB})$ red clump is visible at $J \sim 13$ and $(J-H) \sim 0.52$, with a large magnitude spread due to a combination of differential reddening, metallicity dispersion, and depth effect. Just below the HB red clump, the RGB bump can be seen at $J \sim 13.5$ and $(J-H) \sim 0.52$. The RGB bump is very populated in the high metallicity domain of the bulge, having a large magnitude spread due to the same effects mentioned for the HB clump. The upper RGB, which is brighter than the $\mathrm{HB}$, appears to be wide due to the bulge metallicity dispersion. The bulge main-sequence becomes very broad towards faint magnitudes due to photometric errors, skewing towards red colors. A vertical sequence extending upward $(J \leq 15.5)$ and blueward $(0.15 \leq(J-H) \leq 0.4)$ is due to foreground mainsequence stars belonging to the disk, widely dispersed along the line of sight ( $\mathrm{Ng} \&$ Bertelli 1996). Another vertical sequence corresponding to the disk red clump stars can also be distinguished at $(J-H) \sim 0.5$ for $J<12.5$, dispersed in magnitude as a result of the large spread in distance and reddening (Zoccali et al. 2003). For NGC 6681, a part of the RGB and bright asymptotic giant branch (AGB) stars of the Sgr dSph appears slightly in the CMD to the red side of the RGB sequence of the bulge (Cole 2001; Bellazzini et al. 2003). Fig. 3 also shows a bright portion of the cluster RGB and the blue extension of the HB for each metal-poor bulge cluster.

\section{MEMBER STAR SELECTION}

In order to map the member stars of the bulge target clusters on the sky, it is necessary to minimize the number of contaminating field stars in the photometric information. A simple method is to use an appropriately shaped polygonal mask in a color and magnitude (C-M) space (Grillmair et al. 1995), in which only field stars with color and magnitude that resemble those of cluster stars are counted to optimize the ratio of the cluster stars to field stars. Odenkirchen et al. (2001) introduced an advanced method to improve the statistics of the field star contamination by compressing the observed photometric data in a new C-M space with two orthogonal color indices, from which the weighted sum of field plus cluster stars in photometric data yields the best approximation for the observed total C-M distribution in order to investigate the spatial stellar density distribution around globular clusters.

Following the method of Odenkirchen et al. (2001), we defined C-M masks with new color indices $c_{1}$ and $c_{2}$, which are two orthogonal combination of near-infrared color indices $\left(J-K_{s}\right)$ and $(J-H)$. To derive the new indices, we used stars in the central region of the target clusters, typically located within $2.0 \sim 3.5$ times the half-mass radii $\left(r_{h}\right)$ of the clusters. Indeed, the color-color plots with $\left(J-K_{s}\right)$ and $(J-H)$ indices show that the cluster stars are located in an almost one-dimensional locus, while the $\left(H-K_{s}\right)$ index is less accurate than the others because of decreased sensitivity in the photometric bands. The new indices were then chosen in such a way that $c_{1}$ traces the locus, while $c_{2}$ is perpendicular to it. Eq. 1 presents the rotational transformation of the $\left(J-K_{s}, J-H\right)$ plane into the coordinate system of $\left(c_{1}, c_{2}\right)$,

$$
\begin{gathered}
c_{1}=a\left(J-K_{s}\right)+b(J-H), \\
c_{2}=-b\left(J-K_{s}\right)+a(J-H),
\end{gathered}
$$

where $a$ and $b$ are the transformation coefficients. These coefficients were set by least square fitting of stars in the color-color plane. In columns 2 and 3 of Table 3 , we list the values of coefficients $a$ and $b$ determined for six target clusters. Here, we note that $c_{1}$ contains the systematic variation of color with magnitude, while variations in $c_{2}$ are mainly due to the photometric errors as mentioned in Odenkirchen et al. (2001). In the $\mathrm{C}-\mathrm{M}$ planes of $\left(c_{2}, K_{s}\right)$ and $\left(c_{1}, K_{s}\right)$, the selection of stars was processed to minimize the field star contamination. In the $\left(c_{2}, K_{s}\right)$ plane, we simply applied a magnitude cut to remove faint stars with large photometric error. The limiting magnitude $K_{s}$ has been empirically determined to have $75 \sim 80 \%$ of the photometric completeness according to artificial star tests in the $K_{s}$ band image for each target cluster. The determined limiting magnitudes $K_{s}$ for each cluster are listed in the column 4 of Table 3 .

Next, we applied the C-M mask filtering method (Grillmair et al. 1995) in the $\left(c_{1}, K_{s}\right)$ plane for stars in 

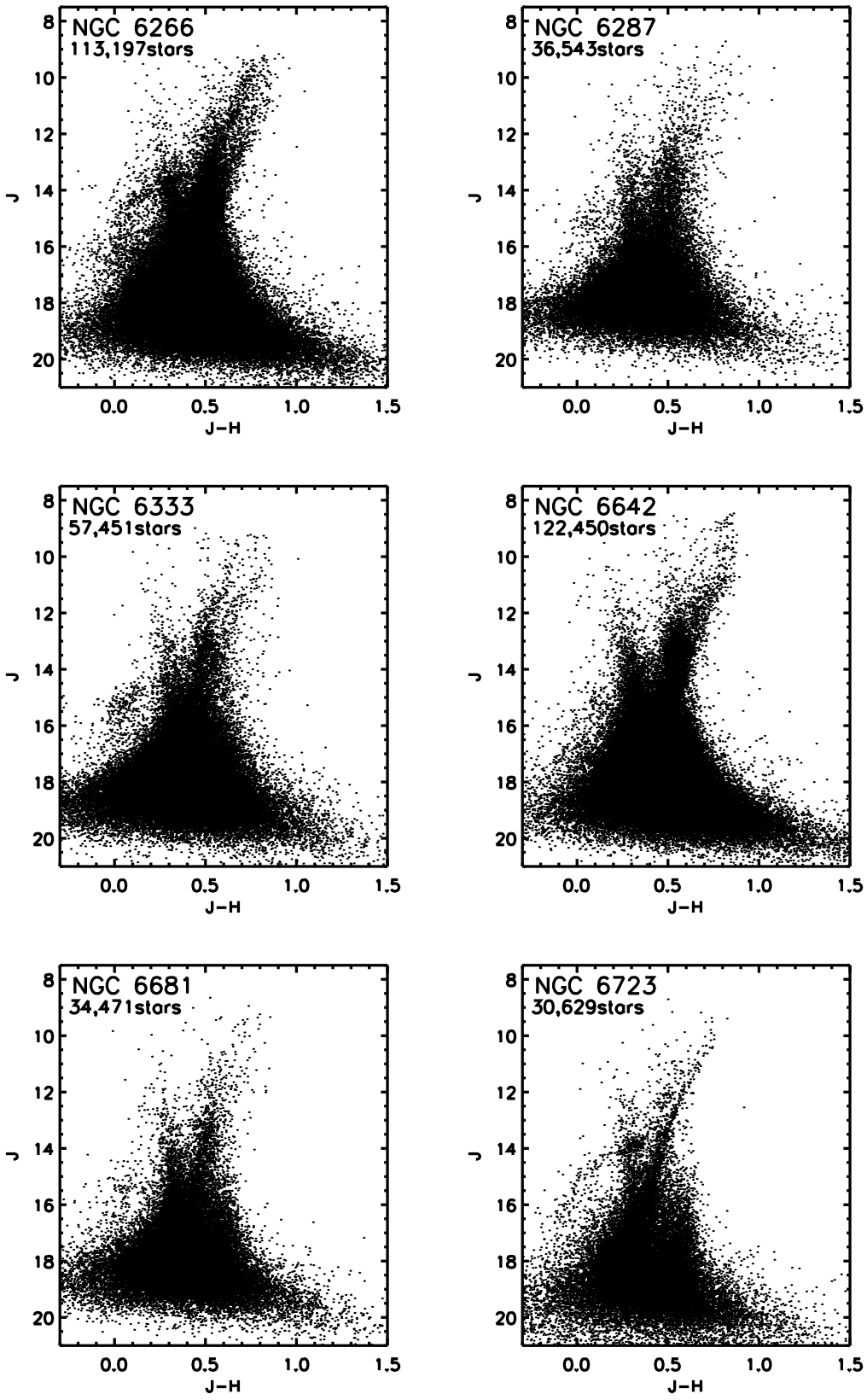

Fig. 3. - Near-infrared $(J-H, J)$ CMDs for stars detected in the observed $\sim 21^{\prime} \times 21^{\prime}$ field of six target clusters. 


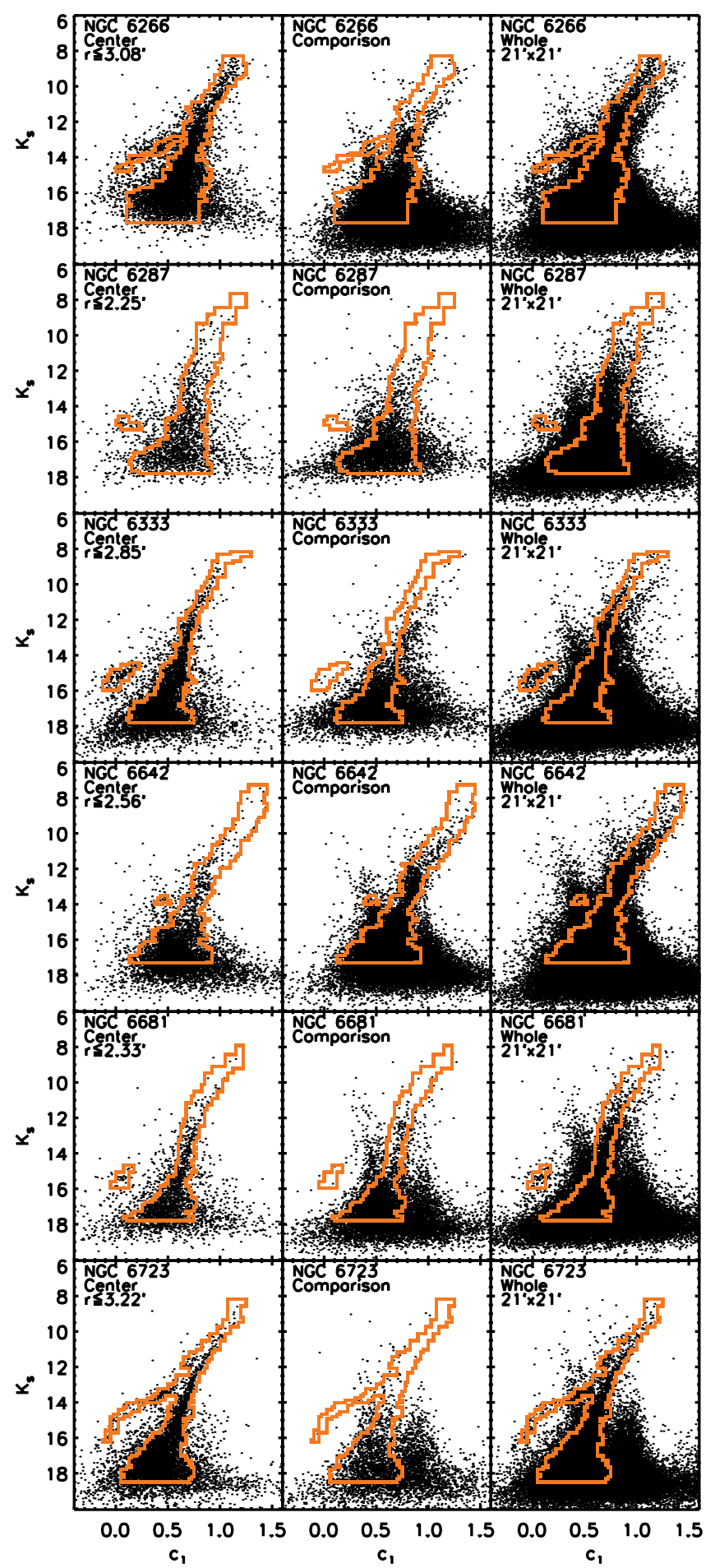

Fig. 4.- $-\left(c_{1}, K_{s}\right)$ CMDs of six target clusters. From left to right, $\left(c_{1}, K_{s}\right)$ planes of the central regions, the comparison fields, and the total observed regions for each cluster are plotted with the mask areas. The boundaries indicate the mask areas determined through the C-M filtering method. 
Table 3.

The coefficients $a$ and $b$ for a transformation from the $\left(J-K_{s}, J-H\right)$ plane to the $\left(c_{1}, c_{2}\right)$ plane, and the limiting magnitude $K_{s}$ of each cluster.

\begin{tabular}{cccc}
\hline \hline Target & $a$ & $b$ & Limiting magnitude $K_{s}$ \\
\hline NGC 6266 & 0.766 & 0.643 & 17.7 \\
NGC 6287 & 0.799 & 0.601 & 17.8 \\
NGC 6333 & 0.787 & 0.617 & 17.8 \\
NGC 6642 & 0.786 & 0.618 & 17.3 \\
NGC 6681 & 0.786 & 0.618 & 17.8 \\
NGC 6723 & 0.788 & 0.616 & 18.5 \\
\hline
\end{tabular}

Table 4.

Background density levels for each cluster, estimated by counting stars within the C-M mask.

\begin{tabular}{cc}
\hline \hline Target & $\begin{array}{c}\text { Background density } \\
(\text { stars/arcmin }\end{array}$ ) \\
\hline NGC 6266 & 73 \\
NGC 6287 & 22 \\
NGC 6333 & 26 \\
NGC 6642 & 81 \\
NGC 6681 & 18 \\
NGC 6723 & 9 \\
\hline
\end{tabular}

the cluster central region and in two comparison fields, which can be seen in the left and middle panels of Fig. 4 for each cluster. The C-M planes of $\left(c_{1}, K_{s}\right)$ were divided into many small sub-grid elements with 0.025 mag width in $c_{1}$ and $0.13 \mathrm{mag}$ height in $K_{s}$. We then measured the number of stars for each sub-grid in the central region of a cluster $n_{c l}\left(c_{1}, K_{s}\right)$ and those in the comparison fields $n_{f}\left(c_{1}, K_{s}\right)$. Assuming that the distribution of field stars does not vary across the observed field, the local signal-to-noise ratio for each sub-grid $s\left(c_{1}, K_{s}\right)$ can be computed by following equation:

$$
s\left(c_{1}, K_{s}\right)=\frac{n_{c l}\left(c_{1}, K_{s}\right)-g n_{f}\left(c_{1}, K_{s}\right)}{\sqrt{n_{c l}\left(c_{1}, K_{s}\right)+g^{2} n_{f}\left(c_{1}, K_{s}\right)}},
$$

where the factor $g$ is the ratio between the cluster central area and the comparison field area. By sorting the elements of $s\left(c_{1}, K_{s}\right)$ into descending order in one dimensional index $k$, we can computed the cumulative number of stars with progressively larger area $a_{k}=k a_{l}$ in the C-M plane. Here, $a_{l}=0.00325 \mathrm{mag}^{2}$ is the area of a single sub-grid element in the $\left(c_{1}, K_{s}\right)$ plane. Denoting $N_{c l}\left(a_{k}\right)$ and $N_{f}\left(a_{k}\right)$ by the cumulative number of stars in the C-M planes of the cluster's central region and the comparison fields, the cumulative signal- to-noise ratio $S\left(a_{k}\right)$ was then computed.

$$
S\left(a_{k}\right)=\frac{N_{c l}\left(a_{k}\right)-g N_{f}\left(a_{k}\right)}{\sqrt{N_{c l}\left(a_{k}\right)+g^{2} N_{f}\left(a_{k}\right)}} .
$$

$S\left(a_{k}\right)$ increases progressively until it reaches a maximum value for a particular sub-grid of the $\left(c_{1}, K_{s}\right)$ plane. The filtering mask in the C-M plane was then determined by selecting all sub-grids with $s\left(c_{1}, K_{s}\right)$ values higher than the maximum of $S\left(a_{k}\right)$. The estimated range of $s\left(c_{1}, K_{s}\right)$ for all six target clusters is between the minimum $\sim 0.45$ and the maximum $\sim 4.67$. All the sub-grids selected in this process, even those with $s\left(c_{1}, K_{s}\right) \leq 1.0$, are assumed to show a high statistical contrast between the surface densities of cluster stars and field stars (cf., Grillmair et al. 1995). Thus, Minor filtering to the sub-grids for RGB stars was also empirically carried out to smooth the mask for each cluster. Finally, the mask was applied to the C-M plane of stars in the whole observed area $\left(\sim 21^{\prime} \times 21^{\prime}\right)$, and stars within the mask are used to analyze the spatial distribution of stars around the target clusters. The solid lines in Fig. 4 represent the boundaries of the selected mask area and the right panels of Fig. 4 show the C-M plane with the mask of selected stars in the total observed field for each target cluster. By extracting stars outside of the masks, about $53 \% \sim 66 \%$ of the total resolved stars were removed as field star contaminants. We note that the mask areas for HB stars were influenced by disk main-sequence stars toward the directions of the target clusters, as shown in Fig. 4. However, the ratios between the selected HB stars and stars in the mask area for each cluster were typically less than 0.01, which suggests that stellar spatial configurations around target clusters are not significantly affected by the selection of HB stars.

\section{SPATIAL DISTRIBUTION OF STARS}

In this section, we examine the features of the stellar spatial configuration around the target clusters through the radial surface density profiles and the Gaussian smoothed two-dimensional iso-density maps of the selected stars. The area coverage $21^{\prime} \times 21^{\prime}$ of the photometric data allows us to investigate the spatial stellar density distribution of the target clusters out to the tidal radius and beyond. Note again that the selected target clusters have tidal radii in the range of $8^{\prime} \sim 10^{\prime}$.

It has long been supposed that globular clusters must a finite edge due to the removal of stars by the Galactic tidal field, and that the radial distribution of stars could be well represented by a King model (King 1966). Nevertheless, Grillmair et al. (1995), in an analysis of star-counts in the outer parts of a few Galactic globular clusters, found extra-cluster overdensity that associated with stars stripped into the Galactic field. Indeed, clusters with evident extratidal overdensity features show a break in the observed radial surface den- 
sity profiles within the tidal radius (Grillmair et al. 1995; Leon et al. 2000; Testa et al. 2000; Rockosi et al. 2002; Lee et al. 2003; Olszewski et al. 2009), which departs from the form predicted by King (1966) models. Numerical simulations also confirmed such a break with power laws in the radial surface density profiles due to the tidal debris around clusters (e.g., Combes et al. 1999; Johnston et al. 1999, 2002).

In the present study, we derived the radial surface density profiles of clusters by applying surface photometry for the inner part and by measuring the number density of stars for the outer parts of the target clusters. For the observed central field $\left(7^{\prime} .7 \times 7^{\prime} .7\right)$ containing the cluster center, we have performed a concentric aperture surface photometry, and present the results from the $H$ images for the target clusters. In the surface photometry, we used concentric annuli of width $9^{\prime \prime}$ divided into eight sectors with an angle of $45^{\circ}$. The local surface brightness $(\mu)$ and the measurement error at each annulus were determined by taking the mean of the eight sector measurements and the standard deviation of the mean. For the measurements of the radial number density profile, we counted all stars in concentric annuli of width $0^{\prime} .5$, and divided the number by the area of each annulus. Only stars pre-selected in the C-M plane, as described in Section 3, have been used for the star counting. We also estimated the mean background density level, by counting stars in the C-M mask of two comparison fields for each target cluster, and then subtracted it from the measured radial number densities. The derived background levels for each cluster are listed in Table 4 . The error of the radial number density profile is based on Poisson statistics from the number of stars counted in each annulus. We note here that star counting is not suitable for measurements of the number density profile in the cluster's central part due to the crowding effect, reducing the surface number densities. Therefore, we combined the dataset of the surface photometry for the inner part with the measured number density profile for the outer part of the cluster, to obtain profiles ranging from the cluster center out to the tidal radius and beyond. The overlapping radial range was empirically adjusted to have similar slopes both in the number density profile and the surface brightness profile. The measured number densities within the inner limit of the overlapped range were not used to analyze the radial surface density profiles, since the crowding effect was not properly corrected for the star counting. In order to combine the two datasets, we adjusted the surface brightness profile for the inner part to the number density profile by using the equation $\log ($ number density $)=-\mu / 2.5+C$, where $C$ is a constant derived separately for each target cluster. We empirically fitted the King (1966) models to the observed surface density profiles over a whole radial range, and checked the overdensity feature by fitting a power law of $r^{\gamma}$ to the outer part of the profiles for each target cluster. We then compared the estimated slope of a power law with previous theoretical and observational results (e.g., Combes et al. 1999; Johnston et al. 1999; Leon et al. 2000; Rockosi et al. 2002; Jordi \& Grebel 2010). Note that we adopted the concentration parameter $c$ for each cluster from Harris (1996) to extract a theoretical King model for the observed radial profile. We mention, however, that the tidal radius of a cluster is no longer listed in the new catalog of Harris (1996) (2010 edition), because it is a model-dependent number and it can also be affected by the existence of tidal structures of escaping stars outside the normal tidal boundary in the King (1966) model.

The configuration of stars around target clusters has been directly examined by constructing the spatial surface iso-density maps for stars pre-selected by the C-M mask filtering method. We first made a twodimensional star count map by measuring the number density of stars in a number of small grids for the observed field. The star count map was then transformed into a surface density map by means of a Gaussian smoothing with various kernel values. The smoothing process enhanced the low spatial frequency of the background structure and removed high spatial frequency variations in the measured star count map. The mean background level and the iso-density levels in a unit of the standard deviation $\sigma$ of the background level were overlaid on the smoothed surface density maps with various kernel values. The directions of the Galactic center, the Galactic plane, and the selected proper motion for each target cluster were also overlaid on the surface density maps in order to compare with the features of the surface iso-density maps.

\subsection{NGC 6266 (M62)}

In Fig. 5, we show the measured radial surface density profile of NGC 6266 . We also overlaid the surface brightness data from the compilation of Trager et al. (1995), which are arbitrarily shifted vertically to the measurements in this study. As shown in Fig. 5, the observed radial surface brightness profile for the inner region $\left(\log \left(r^{\prime}\right) \lesssim 0.4\right)$ is in accordance with previous measurements. We reconfirm, however, that the concentration parameter $c=1.50$ of the King model seems to be significantly lower than the value $c=1.70$ of Harris (1996), as previously indicated by Beccari et al. (2006). The profile we obtained for the outer region does not seem to resemble the data from Trager et al. (1995), most of which were obtained from the photographic or photoelectric observations. Nevertheless, our result is in excellent agreement with high quality photometric data from a wide-field mosaic CCD imaging of Beccari et al. (2006). Apparently, the measured radial surface density profile departs from the King model with a break at $\log \left(r^{\prime}\right) \sim 0.45\left(\approx 2.29 r_{h}, 0.31 r_{t}\right)$. Here, $r_{h}$ and $r_{t}$ indicate the half-mass radius and the tidal radius of the cluster in Harris (1996). The overdensity feature extends out to the tidal radius $r_{t}=$ 


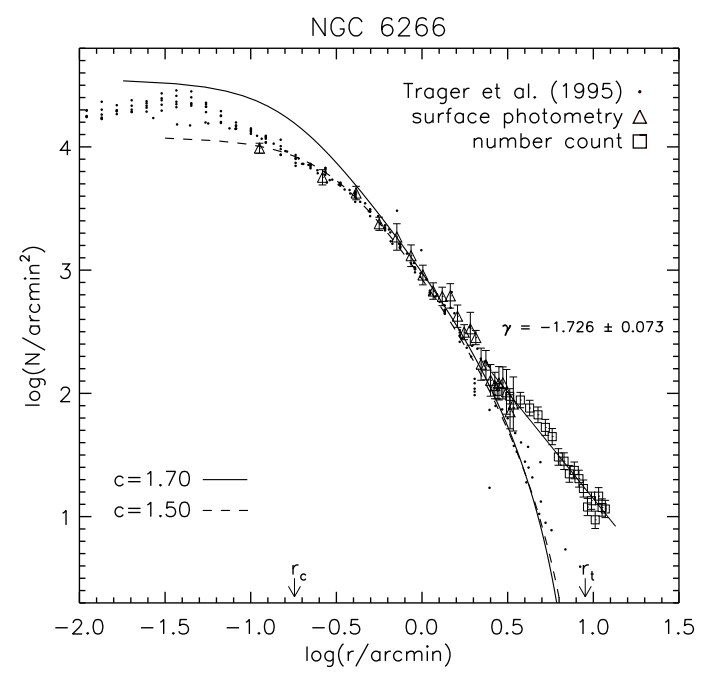

Fig. 5. - Radial surface density profile of NGC 6266 with theoretical King models of $c=1.70$ (solid curve) and $c=1.50$ (dashed curve). Open triangles and squares are data from the surface photometry and the number density measurement, respectively. Data from Trager et al. (1995) are also indicated with dots. The detected overdensity region of the profile $\left(\sim 0.45 \leq \log \left(r^{\prime}\right) \leq \sim 1.07\right)$ is characterized by a power-law with a slope of $\gamma=-1.726 \pm 0.073$, as a straight line. Two arrows represent the core radius and the tidal radius.

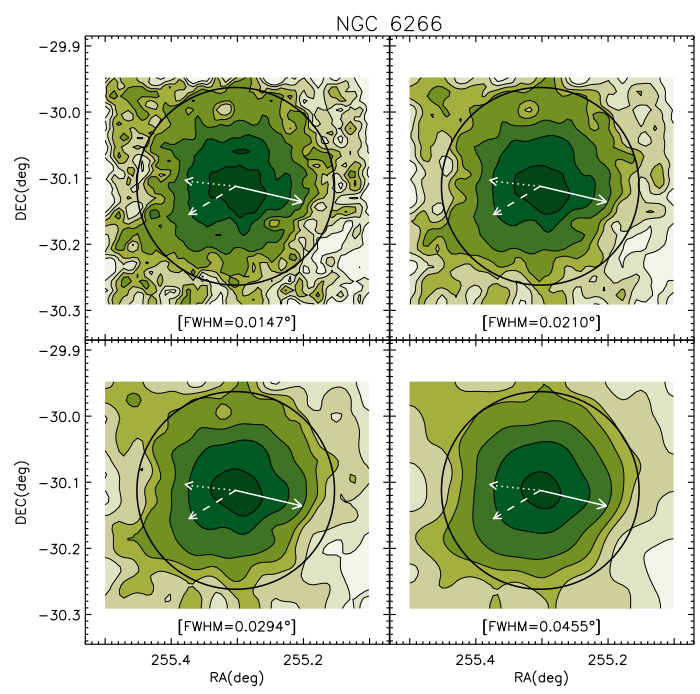

Fig. 6. - Spatial surface iso-density maps around NGC 6266 with Gaussian smoothing kernel values of $0^{\circ} .0147$, $0^{\circ} .0210,0^{\circ} .0294$, and $0^{\circ} .0455$. The circle in the map indicates the tidal radius from Harris (1996). The dotted and dashed arrows display the direction to the Galactic center and the Galactic plane, respectively. The direction of the cluster's proper motion (Dinescu et al. 2003) is indicated by the solid arrow. The iso-density contour levels are the mean background level, $0.25 \sigma, 0.5 \sigma, 0.75 \sigma, 1.25 \sigma, 2.5 \sigma$, and $4 \sigma$ above the mean background level. $8^{\prime} .97$. Instead, the profile in the outer overdensity region $\left(0.45 \lesssim \log \left(r^{\prime}\right) \lesssim 1.07\right)$ resembles a radial power law with a slope of $\gamma=-1.726 \pm 0.073$. The determined slope is significantly steeper than the case of $\gamma=-1$, predicted for a constant mass-loss rate in a cluster over a long time (Johnston et al. 1999), while it is comparable to the value $-1.58 \pm 0.07$ estimated by Rockosi et al. (2002) from the annular averaged densities of stars along the extratidal tails of Pal 5. However, the slope in the overdensity region is shallower than the value of $\gamma=-3$, which is expected in N-body simulations for spatial configuration of stars escaped from a globular cluster (Combes et al. 1999).

A two-dimensional number density map of NGC 6266 was constructed by counting the pre-selected stars in each $0^{\prime} .42 \times 0^{\prime} .42$ size of bin through the observed area. The number density map was then smoothed by a Gaussian filtering with various kernel values of $0^{\circ} .0147$, $0^{\circ} .0210,0^{\circ} .0294$, and $0^{\circ} .0455$. The iso-density contour levels were set to be the measured background level and $0.25 \sigma, 0.5 \sigma, 0.75 \sigma, 1.25 \sigma, 2.5 \sigma$, and $4 \sigma$ above the background level. Fig. 6 shows the smoothed surface density maps of NGC 6266, overlaying the iso-density contour levels. The circle indicates the tidal radius of the cluster. The projected proper motion of the cluster, $\mu_{\alpha} \cos \delta=-3.50 \pm 0.37 \mathrm{mas} \mathrm{yr}^{-1}$ and $\mu_{\delta}=-0.82$

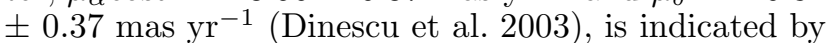
the solid arrow. Dotted and dashed arrows represent the directions of the Galactic center and the Galactic plane, respectively. Fig. 6 clearly shows that a distorted flocculent feature, developed within the tidal radius of the cluster at the contour levels of $0.5 \sigma$ above the background, is in accordance with the overdensity feature on the radial surface density profile of Fig. 5. While there is no strong evidence of a long extended tidal feature in any specific direction, a fairly spherical extratidal halo around the cluster is detected with some asymmetry towards the southeast and the northeast. The marginal extension to the southeast direction is likely to be aligned with the direction of the Galactic plane. A weak extension to the northeast direction at the contour level of $0.75 \sigma$ above the background also corresponds with the opposite direction of the cluster's proper motion, tracing the cluster trailing tail through its orbital path.

\subsection{NGC 6287}

The observed radial surface density profile of NGC 6287, along with the data from Trager et al. (1995), is shown in Fig. 7. The combined profile was fitted with a theoretical King (1966) model of $c=1.60$ (Harris 1996). While the central region of the profile $\left(\log \left(r^{\prime}\right) \lesssim-0.5\right)$ shows a flat plateau because of crowding effect, the King model fitting reveals a break in the slope at $\log \left(r^{\prime}\right) \sim 0.37\left(\approx 3.12 r_{h}, 0.22 r_{t}\right)$. The excess extends out to the tidal radius of the cluster, $r_{t}=10^{\prime} .51$ (Harris 1996). The profile in this overden- 


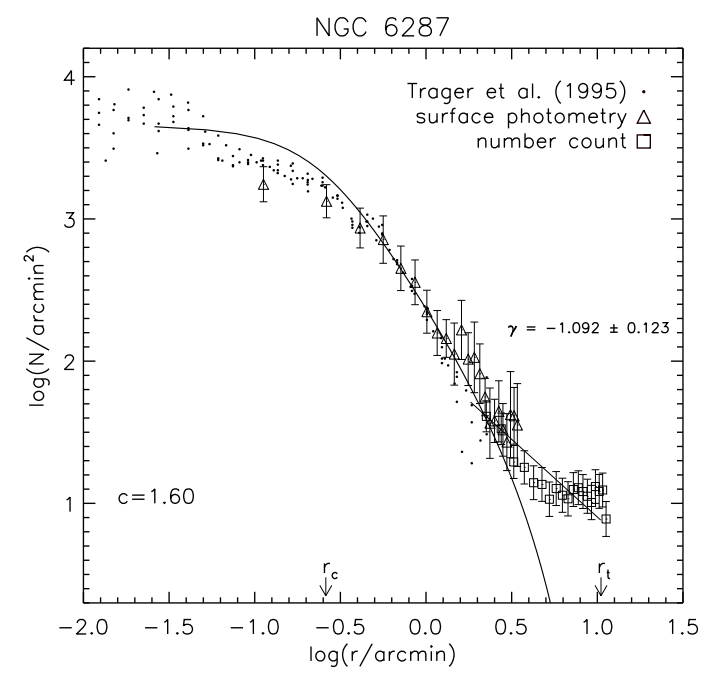

Fig. 7.- Radial surface density profile of NGC 6287 with a theoretical King model of $c=1.60$ (solid curve). Symbols are the same as in Fig. 5. The detected overdensity region of the profile $\left(\sim 0.37 \leq \log \left(r^{\prime}\right) \leq \sim 0.97\right)$ is characterized by a power-law with a slope of $\gamma=-1.092 \pm 0.123$.

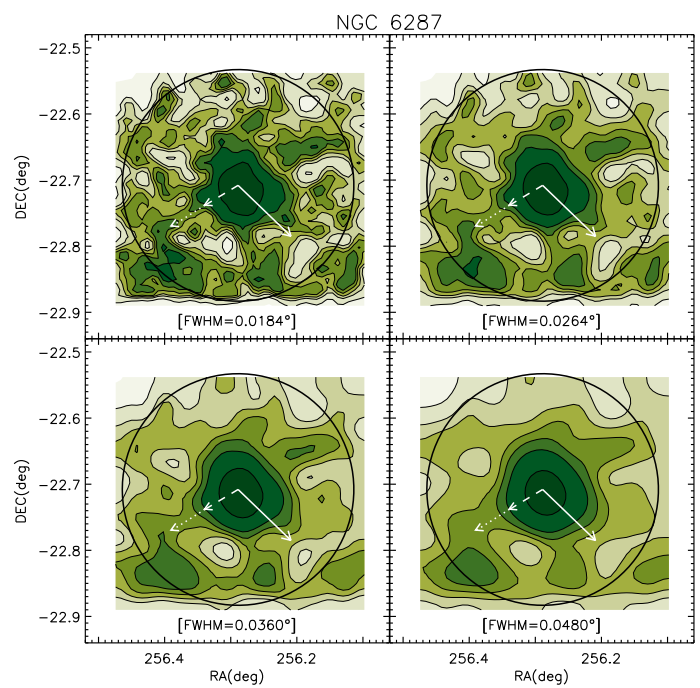

Fig. 8.- Spatial surface iso-density maps of NGC 6287 smoothed by Gaussian kernel values of $0^{\circ} .0184,0^{\circ} .0264$, $0^{\circ} .0360$, and $0^{\circ} .0480$. The circle in the map represents the tidal radius (Harris 1996). The dotted and dashed arrows indicate the direction to the Galactic center and the Galactic plane, respectively. The solid arrow indicates the direction of the cluster's proper motion from Casetti-Dinescu et al. (2010). The iso-density contour levels are the mean background level, $0.6 \sigma, 0.9 \sigma, 1.2 \sigma, 1.5 \sigma, 2 \sigma$, and $4 \sigma$ above the mean background level.

sity region is characterized by a power law with a slope of $\gamma=-1.092 \pm 0.123$, which is comparable to the value of $\gamma=-1$ for the constant mass-loss case of a cluster (Johnston et al. 1999).
In Fig. 8, we present the two-dimensional surface density maps and the overlaid iso-density contours for NGC 6287. To get the surface density maps, we counted the number of stars in each $0^{\prime} .48 \times 0^{\prime} .48$ bin on the C-M mask-selected spatial stellar distribution of NGC 6287, and then smoothed the star count map with Gaussian kernel values of $0^{\circ} .0184,0^{\circ} .0264,0^{\circ} .0360$, and $0^{\circ} .0480$. The iso-density contour levels in the maps are the mean background density, and $0.6 \sigma, 0.9 \sigma, 1.2 \sigma$, $1.5 \sigma, 2 \sigma$, and $4 \sigma$ above the mean background density. It is striking that the contours within the cluster tidal radius show a feature of severe distortion and asymmetry, revealing a complex multiple arm morphology at the level larger than $0.6 \sigma$ above the background. The contours are elongated in the southwest direction of the projected proper motion (solid arrow), $\mu_{\alpha} \cos \delta=-3.68$

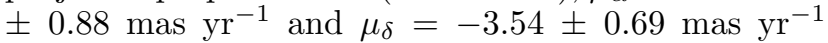
(Casetti-Dinescu et al. 2010), possibly indicating weak evidence of a leading arm of tidal tails. Moreover, the apparent overdensity in the southeast direction, out to the tidal radius, is likely to be aligned with the direction of the Galactic center and plane. The overdensity in the direction of the Galactic anti-center is also observed within the tidal radius of the cluster.

\subsection{NGC 6333 (M9)}

In Fig. 9, we plot the observed radial surface density profile of NGC 6333 . We also overplotted the surface brightness data compiled by Trager et al. (1995). The combined radial profile for the inner part of the cluster is well reproduced by a King model with a concentration parameter $c=1.40$ (cf., Peterson \& Reed 1987). Note that the concentration is significantly higher than the value $c=1.15$ tabulated by Trager et al. (1995). It is apparent in Fig. 9 that the overabundance of member stars is visible as a pronounced break in the outer region of the profile, i.e., $\log \left(r^{\prime}\right) \sim 0.51$ $\left(\approx 3.41 r_{h}, 0.40 r_{t}\right)$. The profile departs from the King model at the break and the overdensity feature extends out to the tidal radius of the cluster. The departure from the King model in the overdensity region can be represented by a power law with a slope of $\gamma=-1.174 \pm 0.069$, which is slightly steeper than the value -1 for the radial profile of the constant mass-loss case (Johnston et al. 1999).

Fig. 10 shows two-dimensional surface stellar density maps around NGC 6333. A raw star count map for the pre-selected stars from C-M mask filtering was first made by counting the number of stars in each $0^{\prime} .42 \times 0^{\prime} .42$ grid-size. We then smoothed the map with various Gaussian kernel values of $0^{\circ} .0147,0^{\circ} .0210$, $0^{\circ} .0315$, and $0^{\circ} .0441$. Iso-density plots are overlaid on the maps with contour levels of the mean background density, and $0.45 \sigma, 0.75 \sigma, 1.05 \sigma, 1.35 \sigma, 2.5 \sigma$, and $5 \sigma$ above the mean background density. There is a severe bias in the southwest corner because of dust extinction by the dark nebula Barnard 64 along the line of sight (see Fig. 17). Nevertheless, the spatial configu- 


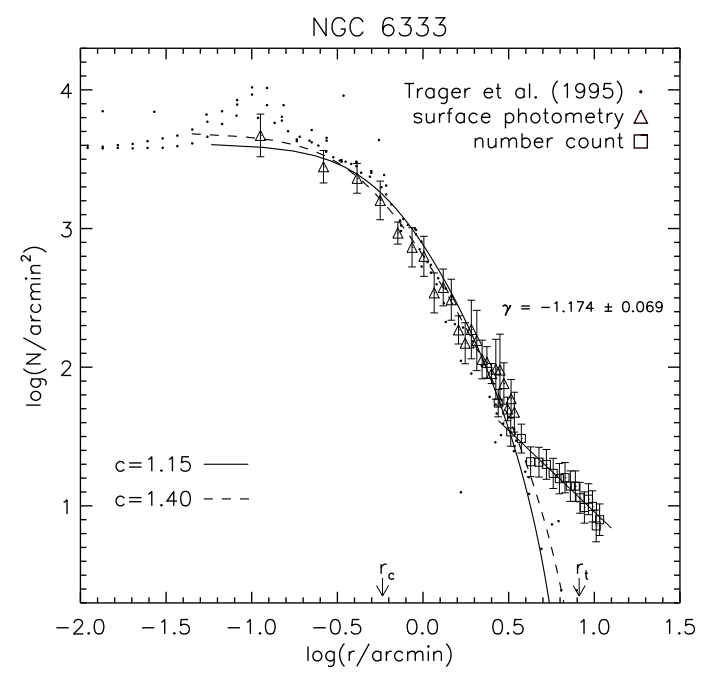

Fig. 9.- Radial surface density profile of NGC 6333 with theoretical King models of $c=1.15$ (solid curve) and $c=1.40$ (dashed curve). Symbols are the same as in Fig. 9. The detected overdensity region of the profile $\left(\sim 0.51 \leq \log \left(r^{\prime}\right) \leq \sim 1.03\right)$ is characterized by a power-law with a slope of $\gamma=-1.174 \pm 0.069$.

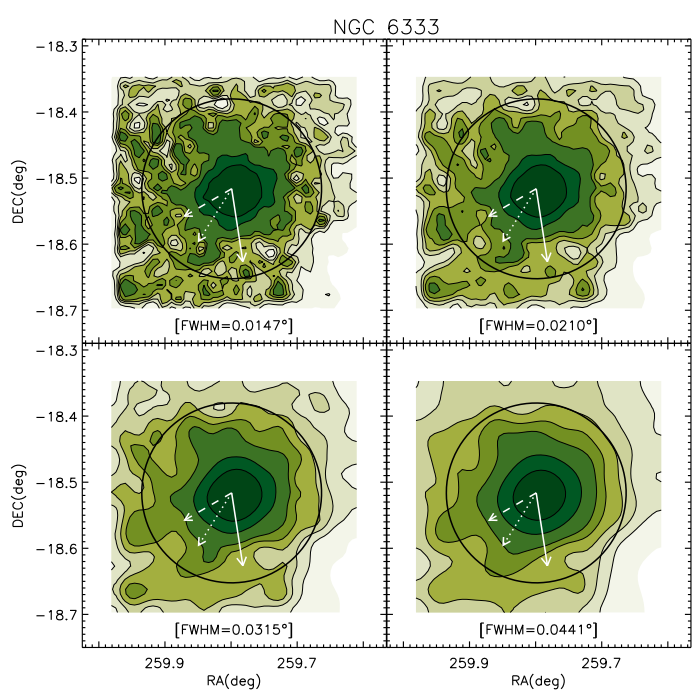

Fig. 10. - Spatial surface iso-density maps around NGC 6333 with Gaussian smoothing kernel values of $0^{\circ} .0147$, $0^{\circ} .0210,0^{\circ} .0315$, and $0^{\circ} .0441$. The circle in the map indicates the tidal radius from Harris (1996). The dotted and dashed arrows represent the direction to the Galactic center and the Galactic plane, respectively. The direction of the cluster's proper motion (Casetti-Dinescu et al. 2010) is represented by the solid arrow. The iso-density contour levels are the mean background level, $0.45 \sigma, 0.75 \sigma, 1.05 \sigma, 1.35 \sigma$, $2.5 \sigma$, and $5 \sigma$ above the mean background level.

ration of stars reveals features of asymmetrically distorted multiple arms within the tidal radius $r_{t}=8^{\prime} .16$ (Harris 1996), and overdensity extensions out to the tidal radius of the cluster. Clearly, extensions toward the southeast direction at the level of $0.75 \sigma$ above the background are associated with the directions of the Galactic center and plane. A weak evidence of extension is also seen toward the direction of the projected proper motion of the cluster, $\mu_{\alpha} \cos \delta=-0.57 \pm 0.57$ mas $\mathrm{yr}^{-1}$ and $\mu_{\delta}=-3.70 \pm 0.50$ mas $\mathrm{yr}^{-1}$ (CasettiDinescu et al. 2010), and its opposite direction, out to the tidal radius of the cluster. No extensions are observed toward the Galactic anti-center in the maps.

\section{$4.4 \quad$ NGC 6642}

The radial surface density profile of NGC 6642 is presented in Fig. 11, along with the compiled data from Trager et al. (1995). An overlaid theoretical King model of $c=1.99$ (Harris 1996) fits well with the observed surface densities for the inner region, excluding the most central region of the cluster $\left(\log \left(r^{\prime}\right) \lesssim-0.6\right)$ because of the crowding effect. The observed profile departs from the King model with a break at $\log \left(r^{\prime}\right) \sim$ $0.01\left(\approx 1.40 r_{h}, 0.10 r_{t}\right)$, extending out to $\log \left(r^{\prime}\right) \sim 0.63$ $\left(\approx 0.42 r_{t}\right)$. The overdensity in this range is characterized by a power law with a slope of $\gamma=-0.843 \pm 0.143$, which is slightly flatter than the case $\gamma=-1$ of a constant mass-loss rate (Johnston et al. 1999). Note that studies of extratidal features from Leon et al. (2000) and Jordi \& Grebel (2010) also show slopes of power law flatter than $\gamma=-1$ in the radial range between $r_{t}$ and $3 r_{t}$ of some clusters. Relatively large errors for the surface densities in this region are attributed to the deviation of the mean for the values of eight sectors. This can be explained by the feature of asymmetric multiple arms, as shown in the surface density maps of Fig. 12. For the outer region $\log \left(r^{\prime}\right) \geq 0.7$, the measured surface densities are likely to remain constant out to the tidal radius of the cluster.

To construct two-dimensional surface density maps around NGC 6642, we first made a star count map for stars selected from the C-M mask filtering by binning the total observed area $\left(\sim 21^{\prime} \times 21^{\prime}\right)$ into small grids of $0^{\prime} .48 \times 0^{\prime} .48$ size. The star count map was then transformed into smoothed surface density maps using a Gaussian filtering algorithm with various kernel values of $0^{\circ} .0160,0^{\circ} .0240,0^{\circ} .0348$, and $0^{\circ} .0440$. Fig. 12 shows the Gaussian smoothed surface density maps of stars around NGC 6642, with overlaid isodensity contour levels of the mean background density, and $0.25 \sigma, 0.4 \sigma, 0.55 \sigma, 0.8 \sigma, 0.93 \sigma$, and $1.3 \sigma$ above the mean background density. It is apparent in Fig. 12 that an overdensity feature larger than $0.8 \sigma$ above the background in the southwest direction extends out to the tidal radius $r_{t}=10^{\prime} .07$ (Harris 1996) of the cluster and beyond. This extension is likely to be aligned with the direction of the Galactic center. The distorted extension to the west direction may correspond to the direction of the Galactic plane. Asymmetric multiple arms and local clumpy overdensity features also ap- 


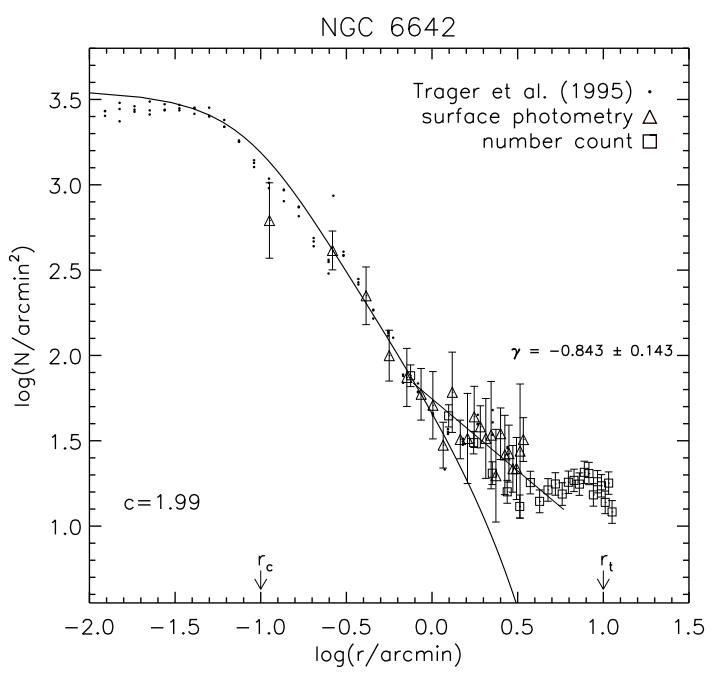

Fig. 11. - Radial surface density profile of NGC 6642 with a theoretical King model of $c=1.99$ (solid curve). Symbols are the same as in Fig. 5. The detected overdensity region of the profile $\left(\sim 0.01 \leq \log \left(r^{\prime}\right) \leq \sim 0.63\right)$ is characterized by a power-law with a slope of $\gamma=-0.843 \pm 0.143$.

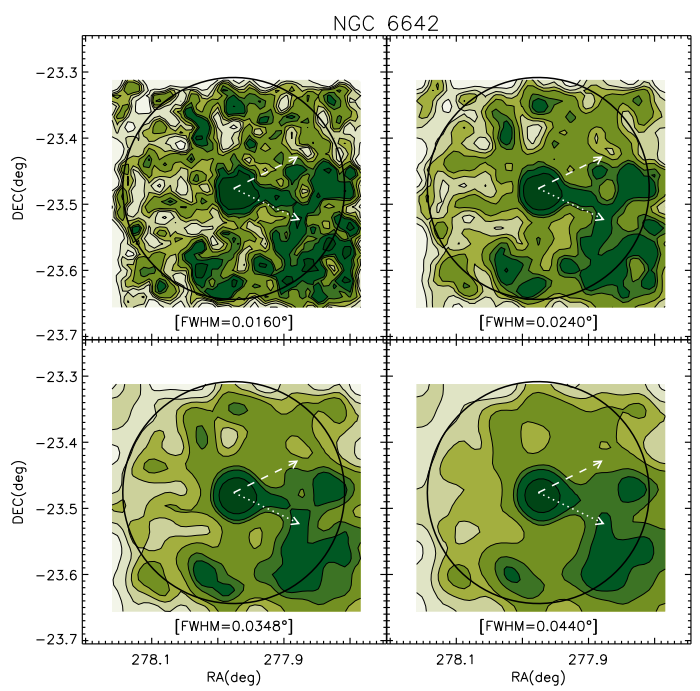

Fig. 12. - Spatial surface iso-density maps of NGC 6642 smoothed by Gaussian kernel values of $0^{\circ} .0160,0^{\circ} .0240$, $0^{\circ} .0348$, and $0^{\circ} .0440$. The circle in the map represents the tidal radius (Harris 1996). The dotted and dashed arrows indicate the direction to the Galactic center and the Galactic plane, respectively. The iso-density contour levels are the mean background level, $0.25 \sigma, 0.4 \sigma, 0.55 \sigma, 0.8 \sigma, 0.93 \sigma$, and $1.3 \sigma$ above the mean background level.

pear within the tidal radius of the cluster. However, we could not refer the cluster orbit from any overdensity structures in the maps, because the proper motion of NGC 6642 has not yet been reported.

\subsection{NGC 6681 (M70)}

In Fig. 13, we plot the radial surface density profile of NGC 6681. The compiled data from Trager et al. (1995) are also plotted with the observed surface densities. The combined profiles are fitted with a theoretical King model of $c=2.50$ (Harris 1996). Note that it is the only post core-collapsed cluster (Djorgovski \& King 1986; Trager et al. 1995) in our sample, showing the highest central concentration in the radial stellar distribution. While the King model does not perfectly fit the observed profile, even in the inner radial range of $-0.6 \lesssim \log \left(r^{\prime}\right) \lesssim-0.4$, the observed profile shows a feature of overdensity, departing from the theoretical model with a break at $\log \left(r^{\prime}\right) \sim 0.51\left(\approx 3.48 r_{h}, 0.41 r_{t}\right)$. The overdensity feature, which is likely to extend out to the cluster's tidal radius $r_{t}=7^{\prime} .91$, can be represented by a power law with a slope of $\gamma=-1.794 \pm 0.297$. The measured slope is significantly steeper than the value of $\gamma=-1$, predicted for a constant mass-loss rate in a cluster over a long time (Johnston et al. 1999), while it is much shallower than the value of $\gamma=-3$ proposed by Combes et al. (1999). However, the measured slope is likely to be comparable to the value $-1.58 \pm 0.07$ estimated by Rockosi et al. (2002) from the annular averaged densities of stars along the extratidal tails of Pal 5.

In Fig. 14, we show Gaussian smoothed two-dimensional surface density maps of stars in the vicinity of NGC 6681. The star count map with $0^{\prime} .6 \times 0^{\prime} .6$ size of grids for the whole observed area $\left(\sim 21^{\prime} \times 21^{\prime}\right)$ was smoothed with various Gaussian kernel values of $0^{\circ} .0160,0^{\circ} .0220,0^{\circ} .0320$, and $0^{\circ} .0418$. The overlaid iso-density contour levels consist of the mean background density, and $0.2 \sigma, 0.4 \sigma, 0.6 \sigma, 1 \sigma, 2 \sigma$, and $6 \sigma$ above the mean background density. In Fig. 14, it is remarkable that the spatial distribution of stars in the vicinity of NGC 6681 shows an extended feature of multiple arms in the outer region within the tidal radius of the cluster. The arms are also extended outside of the cluster's tidal radius $\left(r_{t}=7^{\prime} .91\right.$; Harris 1996) through the observed area. The extension to the northwest direction is directly associated with the direction of the Galactic center and plane. The maps also reveal an overdensity extension to the Galactic anti-center at the east to southeast direction. No proper motion of the cluster has yet been reported for NGC 6681, and then we could not match the cluster's orbit with any extended overdensity structures in the maps, such as those in the directions of the southwest and the north.

\subsection{NGC 6723}

The radial surface brightness values obtained for each annulus at different distances from the center of NGC 6723 are shown in Fig. 15. The compiled data from Trager et al. (1995) were shifted vertically to match up the observed radial profile. The theoreti- 


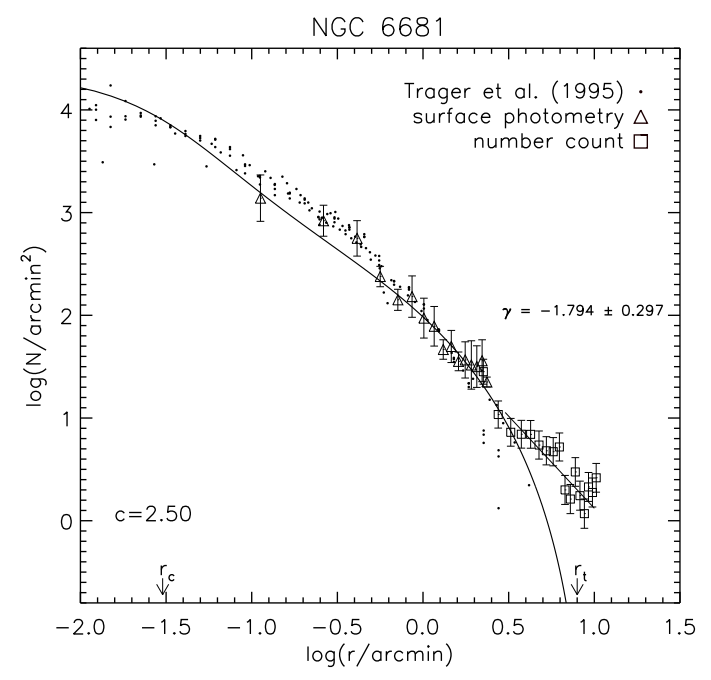

Fig. 13. - Radial surface density profile of NGC 6681 with a theoretical King model of $c=2.50$ (solid curve). Symbols are the same as in Fig. 5. The detected overdensity region of the profile $\left(\sim 0.51 \leq \log \left(r^{\prime}\right) \leq \sim 0.94\right)$ is characterized by a power-law with a slope of $\gamma=-1.794 \pm 0.297$.

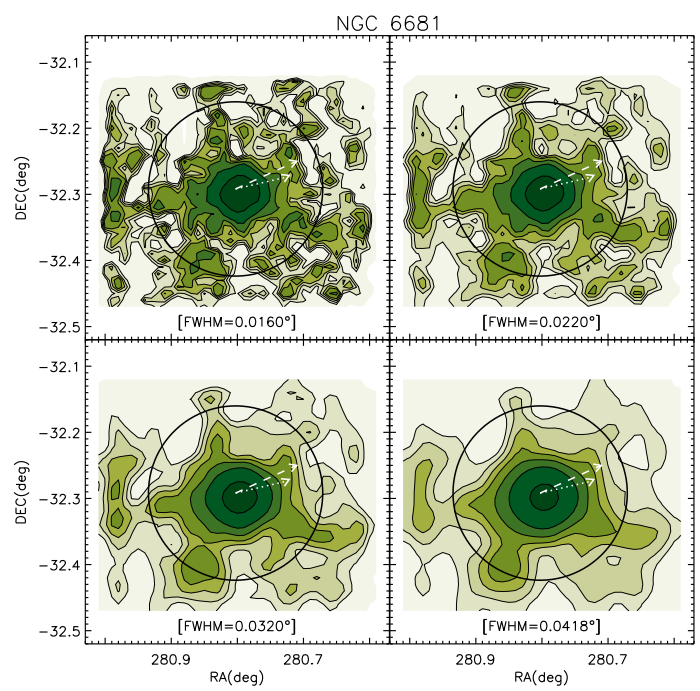

Fig. 14. - Spatial surface iso-density maps around NGC 6681 with Gaussian smoothing kernel values of $0^{\circ} .0160$, $0^{\circ} .0220,0^{\circ} .0320$, and $0^{\circ} .0418$. The circle in the map indicates the tidal radius from Harris (1996). The dotted and dashed arrows represent the direction to the Galactic center and the Galactic plane, respectively. The iso-density contour levels are the mean background level, $0.2 \sigma, 0.4 \sigma, 0.6 \sigma$, $1 \sigma, 2 \sigma$, and $6 \sigma$ above the mean background level.

cal King model of $c=1.05$ (Harris 1996) was empirically fitted to the combined radial profile over the radial range $\log \left(r^{\prime}\right) \lesssim 0.8$. A break in the observed radial surface density profile appears at the radius less than the tidal radius $r_{t}=10^{\prime} .51$ of the cluster (Harris 1996), i.e., $\log \left(r^{\prime}\right) \sim 0.8\left(\approx 3.92 r_{h}, 0.6 r_{t}\right)$. The observed profile departs from the King model at the break, and the overdensity feature extends out to the tidal radius of the cluster and beyond. A power law with a slope of $\gamma=-3.635 \pm 0.293$ is likely to represent the overdensity feature. The determined slope is significantly steeper than the value of $\gamma=-1$ predicted from a constant orbit-averaged mass-loss rate in a cluster (Johnston et al. 1999). Moreover, the slope is much steeper than the value of $\gamma=-3$, which is applicable to the spatial configuration of stars escaped from a globular cluster (Combes et al. 1999). Note also that Jordi \& Grebel (2010) show some clusters with power law slopes larger than -3 in the radial range between $r_{t}$ and $3 r_{t}$.

To examine the two-dimensional spatial configuration of stars in the vicinity of NGC 6723, we first constructed a star count map with a spatial bin size of $0^{\prime} .6 \times 0^{\prime} .6$ for $\sim 21^{\prime} \times 21^{\prime}$ total field of view. We then smoothed the star count map with various Gaussian kernel widths of $0^{\circ} .0140,0^{\circ} .0230,0^{\circ} .0342$, and $0^{\circ} .0440$, yielding the smoothed surface density maps shown in Fig. 16. Iso-density contour levels in Fig. 16 correspond to values of the mean background density, and $0.25 \sigma, 0.5 \sigma, 0.8 \sigma, 2 \sigma, 6 \sigma$, and $14 \sigma$ above the mean background density. The circle indicates the tidal radius $r_{t}=10^{\prime} .51$ of NGC 6723 (Harris 1996). As can be seen in Fig. 16, the spatial stellar distribution is quite symmetrical in the radial range of $r \lesssim 0.6 r_{t}$ at the isodensity levels greater than $0.8 \sigma$ above the background. However, the iso-density contours outer than $\sim 0.6 r_{t}$ show slightly elongated shapes out to the tidal radius of the cluster. This area is associated with the range of the overdensity feature in the radial surface density profile shown in Fig. 15. The east-west direction of the elongated feature is likely to correspond with those of the Galactic center, plane and their opposite directions. In contrast to the elongated feature, extensions in the direction of the proper motion of the cluster, $\mu_{\alpha} \cos \delta=$ $-0.17 \pm 0.45$ mas yr $^{-1}$ and $\mu_{\delta}=-2.16 \pm 0.50$ mas $\mathrm{yr}^{-1}$ (Dinescu et al. 2003), are not clearly revealed in the observed surface density maps of Fig. 16, but are somewhat apparent in the south to southwest direction.

\section{DISCUSSION}

It is worth mentioning that the observed tidal features for each metal-poor cluster can be affected by the intrinsic spatial distribution of background stars, the dust extinction toward the Galactic bulge direction, and background clusters of galaxies in the observed field (e.g., Trager et al. 1995; Chun et al. 2010). Mapping stars in 2MASS catalog located within two $21^{\prime} \times 21^{\prime}$ areas near the clusters NGC 6266 and NGC 6642 , which are the closest objects to the Galactic plane among the target clusters, indicates that the intrinsic background spatial structure is not likely to affect the two-dimensional surface density distribution of stars around the target clusters. Meanwhile, HB 


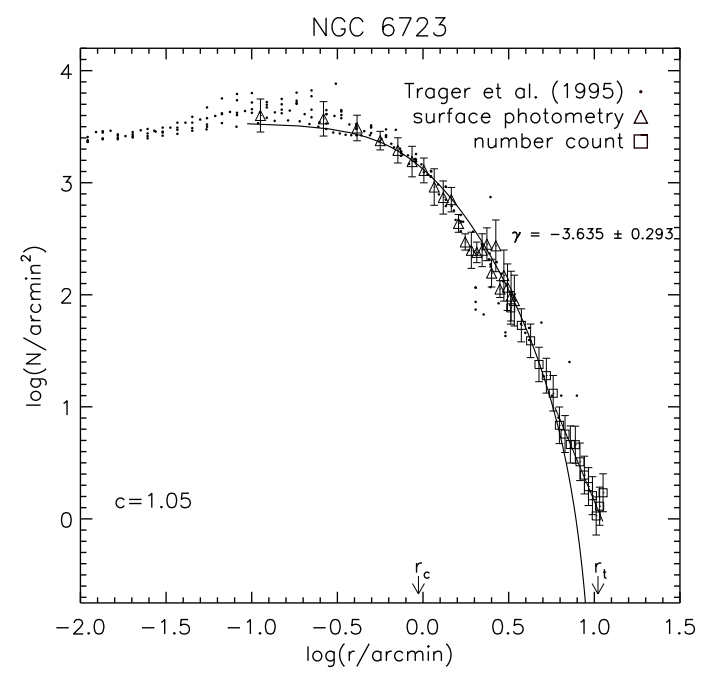

Fig. 15. - Radial surface density profile of NGC 6723 with a theoretical King model of $c=1.05$ (solid curve). Symbols are the same as in Fig. 5. The detected overdensity region of the profile $\left(\sim 0.80 \leq \log \left(r^{\prime}\right) \leq \sim 1.01\right)$ is characterized by a power-law with a slope of $\gamma=-3.635 \pm 0.293$.

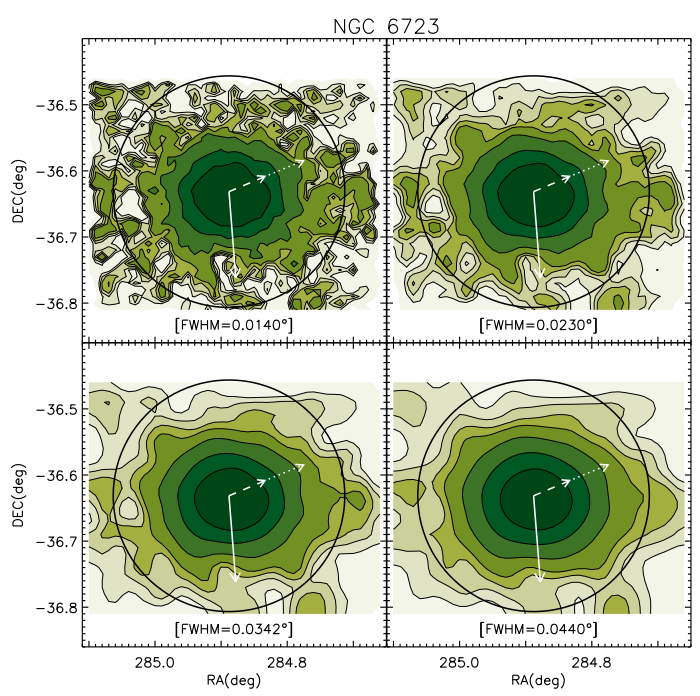

Fig. 16. - Spatial surface iso-density maps of NGC 6723 with Gaussian smoothing kernel values of $0^{\circ} .0140,0^{\circ} .0230$, $0^{\circ} .0342$, and $0^{\circ} .0440$. The circle in the map represents the tidal radius (Harris 1996). The dotted and dashed arrows indicate the direction to the Galactic center and the Galactic plane, respectively. The solid arrow indicates the direction of the cluster's proper motion from Dinescu et al. (2003). The iso-density contour levels are the mean background level, $0.25 \sigma, 0.5 \sigma, 0.8 \sigma, 2 \sigma, 6 \sigma$, and $14 \sigma$ above the mean background level.

stars can also trace the tidal structures of globular clusters, because they are located at a position in a CMD where few field stars are present (e.g., Belokurov et al. 2006a; Jordi \& Grebel 2010). In Fig. 17, we show the
$E(B-V)$ dust extinction maps of the target clusters (Schlegel et al. 1998), overlaying the iso-density contours of the Gaussian smoothed surface density maps with the largest kernel widths in Section 4. Triangles are the extended sources classified in the 2MASS Extended Source Catalog (XSC). Small points indicate the spatial distribution of HB stars that were selected from the C-M mask filtering, as shown in Fig. 4. Here, we discuss individually each cluster for those particular cases that could affect the results for the tidal structure of the target clusters. The ratio $\nu_{\text {tot }} / \nu_{\text {evap }}$ (Gnedin \& Ostriker 1997) has also been used to discuss the dynamical origin of any apparent overdensity extensions in the direction of the Galactic center and plane. The ratio $\nu_{\text {tot }}$ and $\nu_{\text {evap }}$ are the total destruction rate and the evaporation rate of a cluster per Hubble time, respectively.

The cluster NGC 6266 (M62) is placed at $1.7 \mathrm{kpc}$ and $0.9 \mathrm{kpc}$ from the Galactic center and plane, respectively. It is one of the most massive globular clusters $\left(M_{v}=-9.19\right.$; Harris 1996) with a strong EHB (Lee et al. 2007). The extinction map does not exhibit any anticorrelation with the tidally distorted flocculent overdensity feature and the marginal extension in the direction of the Galactic plane, as seen in the surface density maps. It is apparent in Fig. 17 that HB stars do not clearly trace the distorted shape of the iso-density contours for NGC 6266. While the HB stars selected in the C-M mask of the cluster (see in Fig. 4) could contain a considerable number of foreground main sequence stars belonging to the Galactic disk, the abundant number of $\mathrm{HB}$ stars outside the tidal radius may support the hypothesis that the stars are more likely former cluster member stars (Jordi \& Grebel 2010). No background extended sources are found in the outer region of the cluster. The ratio $\nu_{\text {tot }} / \nu_{\text {evap }}=1.0$ from Gnedin \& Ostriker (1997) indicates that the evolution of this cluster is mainly internally driven. The apparent marginal extension on the surface density maps to the Galactic plane then could not be a result of gravitational interaction with the Galactic plane, i.e., a disk shock. Although there is a weak extension in the opposite direction of the cluster proper motion, we should note that Dinescu et al. (2003) suggested that NGC 6266 is a rotationally supported system that belonged to a disk system of the Galaxy, rather than to a pressure-supported system such as the halo.

NGC 6287 is the most metal-poor cluster $([\mathrm{Fe} / \mathrm{H}]=$ -2.05; Harris 1996) in the Galactic bulge $\left(R_{G C}<3\right.$ $\mathrm{kpc})$. It is currently located at distances of $2.1 \mathrm{kpc}$ and $1.8 \mathrm{kpc}$ from the Galactic center and plane, respectively. The dust extinction is very high $(E(B-V) \approx$ $0.6 \sim 0.8)$ toward the cluster. Nevertheless, the complex multiple arm morphology and the elongated extension in the direction of the proper motion seen in the surface iso-density map do not seem to be severely disturbed by the variation of the dust extinction over the observed area. It is difficult to see any correlations between the shape of the iso-density contours and 
the spatial distribution of HB stars, partially because of the small number of selected HB stars. There are no background extended sources in the observed area except those in the cluster central region. The overdensity feature in the direction of the Galactic center and plane indicates that the dynamical evolution of NGC 6287 is currently experiencing the disk- and bulge-shocking events, influenced slightly by the Galactic potential (cf., $\nu_{t o t} / \nu_{\text {evap }}=1.3$, Gnedin \& Ostriker (1997)). It has not yet been clear whether the cluster is likely to be a true nuclear bulge cluster or alternatively a halo object that may have stripped from a dwarf galaxy (e.g., van den Bergh 1993; Stetson \& West 1994; Davidge \& Courteau 1999; Davidge 2001). In a recent proper motion study for globular clusters in the inner Milky Way, Casetti-Dinescu et al. (2010) associated the cluster NGC 6287 with the halo rather than the bar of the Galaxy.

The cluster NGC 6333 (M9) is located at distances of $1.7 \mathrm{kpc}$ and $1.5 \mathrm{kpc}$ from the Galactic center and plane, respectively. The presentation of the overdensity features around this cluster in the iso-density maps illustrates a strong background fluctuation in the southwest direction, owing to the dust extinction along the line of sight. Indeed, as clearly traced by the extinction map in Fig. 17, the southwest field of the observed area was polluted by the dark nebula Barnard 64 located at $r \sim 30^{\prime}$ west of NGC 6333. Since this induced a strong dust extinction over $E(B-V) \sim 0.7$, it was difficult to detect any tidal features on the stellar spatial distribution to the direction. While most of the HB stars are within the innermost area of the cluster, only a few of these are located in the outer region of the cluster and outside of its tidal radius. The apparent distribution of $\mathrm{HB}$ stars is not likely to trace the overdensity features on the iso-density map. Considering only two outer extended sources, located at the northwest corner of the observed area beyond the tidal radius, we found no correlation between the spatial configurations of the background galaxies and the observed stars around NGC 6333. The apparent overdensity extensions toward the Galactic center and plane may due to a strong influence of the Galaxy on this cluster in the forms of disk shock and bulge shock, as indicated by the ratio $\nu_{t o t} / \nu_{\text {evap }}=5.1$ (Gnedin \& Ostriker 1997). Note that NGC 6333 has an uncertain membership between the halo and the thick disk due to the distance uncertainty, in spite of accurate measurements of the space velocity and absolute proper motion of the cluster (Casetti-Dinescu et al. 2010).

NGC 6642 is a globular cluster located at distances of $1.7 \mathrm{kpc}$ and $0.9 \mathrm{kpc}$ from the Galactic center and plane. It has a rather high concentration $c=1.99$ (Harris 1996) of its dynamical structure. Trager et al. (1995) classified the cluster as a possibly core-collapsed cluster. From the photometric study for NGC 6642 with the ACS/WFC system of the HST, Balbinot et al. (2009) also found that the radial density profile shows no evidence of a well-resolved core, corroborat- ing the idea that NGC 6642 is a core-collapsed cluster. It is apparent in Fig. 17 that the dust extinction map does not show any distinctive anticorrelations with the overdensity features revealed on the iso-density contour map, such as distorted extensions toward the Galactic center and plane, asymmetric multiple arm morphology, and several local clumpy structures. The detected HB stars are spread out rather broadly throughout the observed area. Nevertheless, the fact that many HB stars in the field are found in the overdensity regions in the directions of the Galactic center and plane indicates that the spatial distribution of HB stars is likely to trace the tidally distorted dynamical structure of the cluster. We caution, however, that a considerable number of disk main sequence stars can be included in the sample of $\mathrm{HB}$ stars, which were selected in the C-M mask of the cluster (see Fig. 4). Note also that no background extended sources are found in the observed area, except for one source in the cluster's center. The overdensity features to the Galactic center and plane could be a result of a strong gravitational interaction with the Galaxy, as indicated by the ratio $\nu_{\text {tot }} / \nu_{\text {evap }}=8.3$ (Gnedin \& Ostriker 1997). Indeed, Balbinot et al. (2009) found that the present-day mass function of NGC 6642 reflects the disk and bulge shocking through its current perigalacticon passage. They also expected the presence of a tidal tail around the young halo cluster NGC 6642, as a result of depletion of low-mass stars.

NGC 6681 (M70) is placed at distances of $2.1 \mathrm{kpc}$ and $1.9 \mathrm{kpc}$ from the Galactic center and plane. The cluster has been classified as a core-collapsed globular cluster (Djorgovski \& King 1986; Trager et al. 1995) with the highest value of the dynamical concentration $c=2.5$ (Harris 1996). It is also known as a cluster with a moderate EHB morphology (Lee et al. 2007). While the proper motion has not been reported yet, Pryor et al. (1989) suggested that this cluster is more likely a member of the halo population, based on its low metallicity and high radial velocity of $218.7 \pm 1.2 \mathrm{~km}$ $\mathrm{s}^{-1}$. As shown in Fig. 17, the dust extinction toward the cluster is relatively low, with a small variation over the observed area, so that the observed overall overdensity features, such as multiple arms at the outer region of the cluster and extensions in the iso-density map, do not seem to be disentangled from the dust extinction. Indeed, extensions toward directions of the Galactic center, anticenter, and plane might be the result of the dynamical interaction with the Galaxy in the form of disk and bulge shocks, as indicated by the ratio $\nu_{\text {tot }} / \nu_{\text {evap }}=2.3$ (Gnedin \& Ostriker 1997). Apparently, the distribution of $\mathrm{HB}$ stars is not directly connected to the tidally distorted dynamical structure of the cluster. However, a few HB stars outside the cluster could be likely former cluster member stars. It is difficult to conclude that the background galaxies are associated with any apparent extensions in the iso-density map, because only one extended source is located in the observed area. 

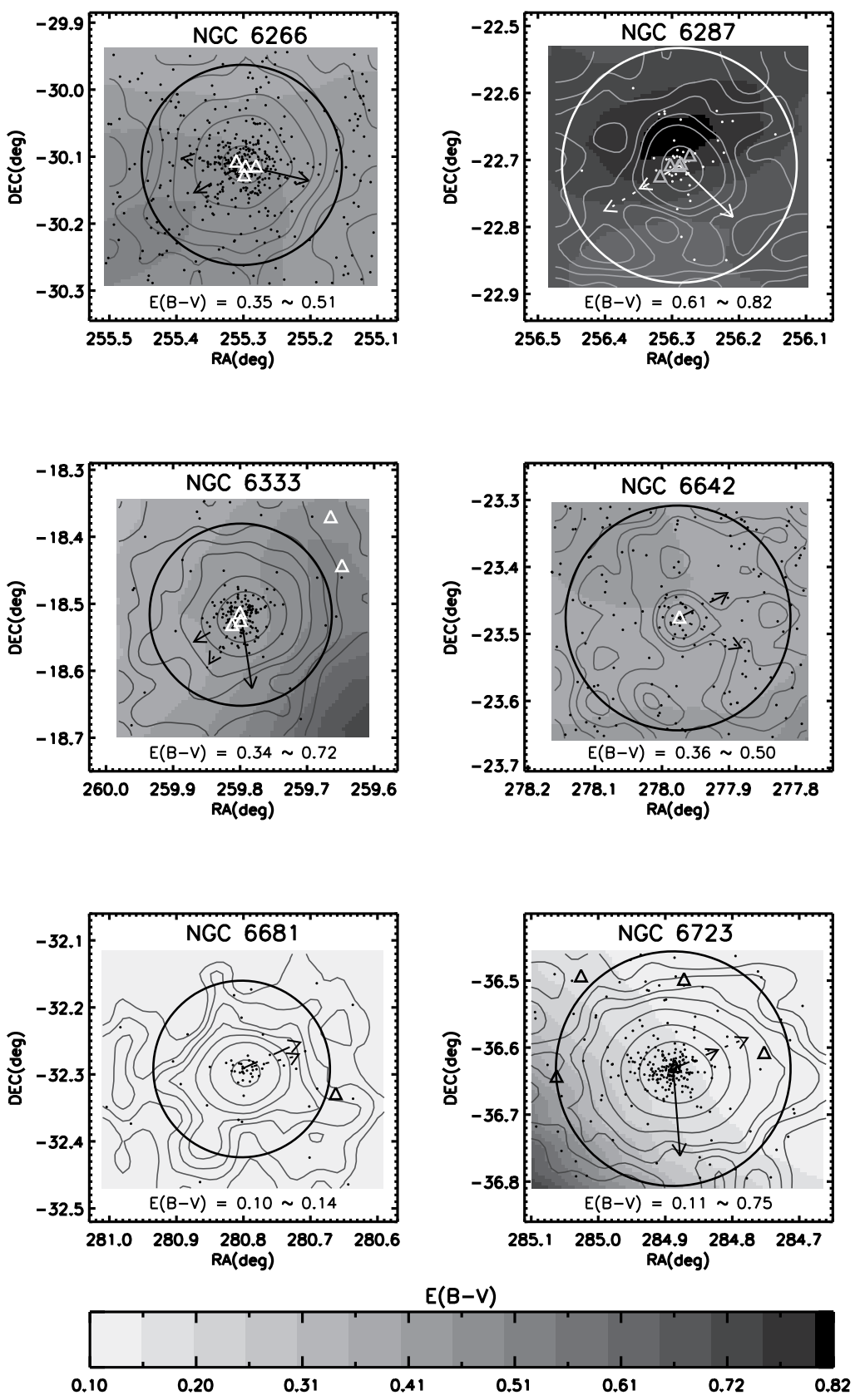

Fig. 17.- The $E(B-V)$ dust extinction maps of the six target clusters (Schlegel et al. 1998). The iso-density contours of the Gaussian smoothed surface density maps with the largest kernel widths in Section 4 are overlaid on the map. Circles indicate the tidal radii of the clusters. The dotted and dashed arrows show the direction to the Galactic center and the Galactic plane, respectively. The direction of the cluster's proper motion (Dinescu et al. 2003; Casetti-Dinescu et al. 2010) is indicated by the solid arrow. Triangles are the extended sources identified in 2MASS Extended Source Catalog. The small points on the maps are HB stars, which were selected from the C-M mask filtering. 
NGC 6723, which is located at distances $2.6 \mathrm{kpc}$ both from the Galactic center and plane, is a cluster with a relatively low concentration $c=1.05$ (Harris 1996) of its dynamical structure. The cluster has a moderate EHB morphology (Lee et al. 2007). Dinescu et al. (2003) reported that the cluster has kinematics consistent with halo membership and location and velocity components that produce a highly inclined orbit. Although there is a local higher extinction in the southeast corner of the observed field, the overall extinction map does not induce any biases to the elongated overdensity features in the east-west direction on the observed surface iso-density map. The distribution of the selected HB stars also seems to follow the elongated shape of the iso-density map. We note, however, that this is only suggestive rather than conclusive, due to the small number of HB stars in the outer part of the cluster. Except for one source in the central region of the cluster, the positions of four background extended sources seem not to be directly connected with the elongated shape of the iso-density contours. Instead, they are likely correlated with the clumpy overdensity feature at the positions on the surface iso-density map of Fig. 16. As suggested by Gnedin \& Ostriker (1997), the elongated overdensity feature of the cluster can be explained by the evolution of the cluster driven by the Galaxy, such as bulge and disk shocking processes, with the ratio $\nu_{t o t} / \nu_{\text {evap }}=2.0$.

\section{CONCLUSION}

We have used wide-field $\left(\sim 21^{\prime} \times 21^{\prime}\right)$ near-infrared $J, H$, and $K_{s}$ images of six metal-poor $([\mathrm{Fe} / \mathrm{H}]<-1.0)$ globular clusters in the Galactic bulge $\left(R_{G C}<3 \mathrm{kpc}\right)$, namely NGC 6266, NGC 6287, NGC 6333, NGC 6642, NGC 6681, and NGC 6723, to search for the clusters' tidal features on the spatial stellar distribution. The cluster's member star candidates were selected by applying a C-M mask filtering algorithm to the nearinfrared color-magnitude diagrams of stars detected in the observed area. The radial surface density profiles have been obtained from the surface photometry of the cluster's images and the star counting to the photometric data of the selected stars. Compared with the theoretical King models, the radial surface density profiles of all the target clusters showed the overdensity features with a break inside the tidal radius. The detected break points were at $1.5 r_{h} \sim 4.0 r_{h}$ of the target clusters, which indicates that the crowding effect in the overdensity regions could not seriously affect to determine the break points in the radial profiles. The profiles in the overdensity region have been characterized by a radial power law. The smoothed two-dimensional surface density maps revealed that all the clusters show distorted asymmetric iso-density contours at the radial range, and an the overdensity feature in the radial surface density profiles. Apparently, the observed tidal overdensity features are likely to be associated with the effects of the dynamical interaction of the Galaxy and cluster space motions. However, we note that deeper imaging data than IRSF are necessary in order to detect fine stellar substructures which is not obvious in this study and to understand the dynamical interaction between the Galaxy and clusters, because lowmass stars below thee turn-off point were stripped from globular clusters and finally form the stellar substructures (Combes et al. 1999).

The comparison of our results to the theoretical study of the globular cluster destruction rates by Gnedin \& Ostriker (1997) shows generally good agreement (cf., Jordi \& Grebel 2010). Among the six metalpoor Galactic bulge clusters in our sample, five of them (i.e., NGC 6287, NGC 6333, NGC 6642, NGC 6681, and NGC 6723$)$, with destruction rate $\left(\nu_{\text {tot }} / \nu_{\text {evap }}\right)$ ratio higher than the value 1 , showed a tidal overdensity structure stretching into the direction of the Galactic center or plane, as a possible consequence of the bulge and disk shock of the Galaxy. Indeed, the kinematics of the five metal-poor clusters (e.g., Dinescu et al. 2003; Casetti-Dinescu et al. 2010) also indicate that they likely belong to a halo system, currently passing through the Galactic bulge and experiencing a strong interaction with the Galaxy. For the exceptional cluster NGC 6266, the observed tidal structure might not be suffering a strong shock, as confirmed by the ratio $\nu_{\text {tot }} / \nu_{\text {evap }}=1$, which indicates that the dynamical evolution of the cluster is mainly internally driven. This cluster also has kinematics consistent with the disk membership (Dinescu et al. 2003).

These observational results of tidal overdensity features in the configuration of stars around target globular clusters would support the accretion scenario that the old stellar system of the Galactic bulge was formed by the mergers of primordial building blocks and that several metal-poor clusters in the Galactic bulge are the surviving remnants of them. However, it should be noted that data for a complete set of space motions of globular clusters in the Galactic bulge and the confirmation of the spatially aligned shape of bulge globular clusters through kinematical properties are essential to verify their association with the formation of the stellar system of the Galactic bulge, and to provide strong evidence for the hierarchical merging scenario. In addition, recent observations indicate that some of the metal-rich clusters could have also survived in the Galactic bulge as merger remnants (e.g., Ferraro et al. 2009). Thus, further deep and wide-field photometric observations for additional metal-poor and metal-rich globular clusters in the Galactic bulge are required to provide more accurate observational constraints of the large scale configuration of stars around clusters.

\section{ACKNOWLEDGMENTS}

This research was supported by the Basic Science Research Program through the National Research Foundation of Korea (NRF) funded by the Ministry of Education, Science and Technology (2013R1A1A2006826). 
This work is partially supported by the KASI-Yonsei Joint Research Program for the Frontiers of Astronomy and Space Science funded by the Korea Astronomy and Space Science Institute.

\section{REFERENCES}

Aguerri, J. A. L., Balcells, M., \& Peletier, R. F. 2001, Growth of Galactic Bulges by Mergers. I. Dense Satellites, A\&A, 367, 428

Balbinot, E., Santiago, B. X., Bica, E., \& Bonatto, C. 2009, The Globular Cluster NGC 6642: Evidence for a Depleted Mass Function in a Very Old Cluster, MNRAS, 396, 1596

Baugh, C. M., Cole, S., \& Frenk, C. S. 1996, Evolution of the Hubble Sequence in Hierarchical Models for Galaxy Formation, MNRAS, 283, 1361

Beccari, G., Ferraro, F. R., Possenti, A., Valenti, E., Origlia, L., \& Rood, R. T. 2006, The Dynamical State and Blue Straggler Popullation of the Globular Cluster NGC 6266 (M62), AJ, 131, 2551

Bedin, L. R., Piotto, G., Anderson, J., Cassisi, S., King, I. R., Momany, Y., \& Carraro, G. 2004, $\omega$ Centauri: The Population Puzzle Goes Deeper, ApJ, 605, L125

Bellazzini, M., Ibata, R., Ferraro, F. R., \& Testa, V. 2003, Tracing the Sgr Stream with 2MASS. Detection of Stream Stars around Outer Halo Globular Clusters, A\&A, 405, 577

Belokurov, V., Evans, N. W., Irwin, M. J., Hewett, P. C., \& Wilkinson, M. I. 2006a, The Discovery of Tidal Tails around the Globular Cluster NGC 5466, ApJ, 637, L29

Belokurov, V., et al. 2006b, The Field of Streams: Sagittarius and Its Siblings, ApJ, 642, L137

Belokurov, V., et al. 2007a, The Hercules-Aquila Cloud, ApJ, 657, L89

Belokurov, V., et al. 2007b, An Orphan in the "Field of Streams", ApJ, 658, 337

Bica, E., Bonatto, C., Barbuy, B., \& Ortolani, S. 2006, Globular Cluster System and Milky Way Properties Revisited, A\&A, 450, 105

Capuzzo-Dolcetta, R., Di Matteo, P., \& Miocchi, P. 2005, Formation and Evolution of Clumpy Tidal Tails around Globular Clusters, AJ, 129, 1906

Casetti-Dinescu, D. I., Girard, T. M., Korchagin, V. I., van Altena, W. F., \& López, C. E. 2010, Space Velocities of Southern Globular Clusters. VI. Nine Clusters in the Inner Milky Way, AJ, 140, 1282

Chun, S.-H., et al. 2010, A Wide-Field Photometric Survey for Extratidal Tails Around Five Metal-Poor Globular Clusters in the Galactic Halo, AJ, 139, 606

Chun, S.-H., Kim, J.-W., Kim, M.-J., Kim, H.-I., Park, J.-H., \& Sohn, Y.-J. 2012, A Feature of Stellar Density Distribution within the Tidal Radius of Globular Cluster NGC 6626 (M28) in the Galactic Bulge, AJ, 144, 26
Cole, A. A. 2001, The 2MASS Color-Magnitude Diagram of the Center of the Sagittarius Dwarf Galaxy. Photometric Measurements of a Surprisingly High Mean Metallicity, ApJ, 559, L17

Combes, F., Leon, S., \& Meylan, G. 1999, N-Body Simulations of Globular Cluster Tides, A\&A, 352, 149

Côté, P. 1999, Kinematics of the Galactic Globular Cluster System: New Radial Velocities for Clusters in the Direction of the Inner Galaxy, AJ, 118, 406

Côté, P., Marzke, R. O., West, M. J., \& Minniti, D. 2000, Evidence for the Hierarchical Formation of the Galactic Spheroid, ApJ, 533, 869

D’Antona, F., Bellazzini, M., Caloi, V., Pecci, F. F., Galleti, S., \& Rood, R. T. 2005, A Helium Spread among the Main-Sequence Stars in NGC 2808, ApJ, 631,868

Davidge, T. J. 2001, Near-Infrared Imaging of the Central Regions of Metal-Poor Inner Spheroid Globular Clusters, AJ, 121, 3100

Davidge, T. J., \& Courteau, S. 1999, High Angular Resolution JHK Imaging of the Centers of the MetalPoor Globular Clusters NGC 5272 (M3), NGC 6205 (M13), NGC 6287, and NGC 6341 (M92), AJ, 117, 1297

Dinescu, D. I., Girard, T. M., van Altena, W. F., \& López, C. E. 2003, Space Velocities of Southern Globular Clusters. IV. First Results for Inner Galaxy Clusters, AJ, 125, 1373

Djorgovski, S., \& King, I. R. 1986, A Preliminary Survey of Collapsed Cores in Globular Clusters, ApJ, 305, L61

Fall, S. M., \& Zhang, Q. 2001, Dynamical Evolution of the Mass Function of Globular Star Clusters, ApJ, 561,751

Ferraro, F. R., et al. 2009, The Cluster Terzan 5 as a Remnant of a Primordial Building Block of the Galactic Bulge, Nature, 462, 483

Gnedin, O. Y., \& Ostriker, J. P. 1997, Destruction of the Galactic Globular Cluster System, ApJ, 474, 223

Grillmair, C. J. 2006, Detection of a $60^{\circ}$-Long Dwarf Galaxy Debris Stream, ApJ, 645, L37

Grillmair, C. J. 2009, Four New Stellar Debris Streams in the Galactic Halo, ApJ, 693, 1118

Grillmair, C. J., \& Dionatos, O. 2006, Detection of a $63^{\circ}$ Cold Stellar Stream in the Sloan Digital Sky Survey, ApJ, 643, L17

Grillmair, C. J., Freeman, K. C., Irwin, M., \& Quinn, P. J. 1995, Globular Clusters with Tidal Tails: Deep Two-Color Star Counts, AJ, 109, 2553

Grillmair, C. J., \& Johnson, R. 2006, The Detection of a $45^{\circ}$ Tidal Stream Associated with the Globular Cluster NGC 5466, ApJ, 639, 17 
Grillmair, C. J., Ajhar, E. A., Faber, S. M., Baum, W. A., Holtzman, J. A., Lauer, T. R., Lynds, C. R., \& O’Neil, E. J. Jr. 1996, Hubble Space Telescope Observations of Globular Clusters in M31. II. Structural Parameters, AJ, 111, 2293

Han, S.-I., Lee, Y.-W., Joo, S.-J., Sohn, S. T., Yoon, S.-J., Kim, H. S., \& Lee, J.-W. 2009, The Presence of Two Distinct Red Giant Branches in the Globular Cluster NGC 1851, ApJ, 707, L190

Harris, W. E. 1996, A Catalog of Parameters for Globular Clusters in the Milky Way, AJ, 112, 1487

Ibata, R. A., Gilmore, G., \& Irwin, M. J. 1994, A Dwarf Satellite Galaxy in Sagittarius, Nature, 370, 194

Ibata, R. A., Gilmore, G., \& Irwin, M. J. 1995, Sagittarius: the Nearest Dwarf Galaxy, MNRAS, 277, 781

Ibata, R., Irwin, M., Lewis, G. F., \& Stolte, A. 2001, Galactic Halo Substructure in the Sloan Digital Sky SUrvey: The Ancient Tidal Stream from the Sagittarius Dwarf Galaxy, ApJ, 547, L133

Ibata, R. A., Wyse, R. F. G., Gilmore, G., Irwin, M. J., \& Suntzeff, N. B. 1997, The Kinematics, Orbit, and Survival of the Sagittarius Dwarf Spheroidal Galaxy, AJ, 113, 634

Ivezić, Z̆., et al. 2000, Candidate RR Lyrae Stars Found in Sloan Digital Sky Survey Commissioning Data, AJ, 120, 963

Johnston, K. V., Choi, P. I., \& Guhathakurta, P. 2002, Interpreting the Morphology of Diffuse Light around Satellite Galaxies, AJ, 124, 127

Johnston, K. V., Sigurdsson, S., \& Hernquist, L. 1999, Measuring Mass-Loss Rates from Galactic Satellites, MNRAS, 302, 771

Jordi, K., \& Grebel, E. K. 2010, Search for Extratidal Features around 17 Globular Clusters in the Sloan Digital Sky Survey, A\&A, 522, 71

Jurić, M., et al. 2008, The Milky Way Tomography with SDSS. I. Stellar Number Density Distribution, ApJ, 673, 864

King, I. R. 1966, The Structure of Star Clusters. III. Some Simple Dynamical Models, AJ, 71, 64

Lauchner, A., Powell, W. L. J., \& Wilhelm, R. 2006, Discovery of a Tidal Stream Extending from NGC 5053, ApJ, 651, L33

Law, D. R., \& Majewski, S. R. 2010, Assessing the Milky Way Satellites Associated with the Sagittarius Dwarf Spheroidal Galaxy, ApJ, 718, 1128

Lee, Y.-W., Gim, H. B., \& Casetti-Dinescu, D. I. 2007, Kinematic Decoupling of Globular Clusters with the Extended Horizontal Branch, ApJ, 661, L49

Lee, Y.-W., Joo, J.-M., Sohn, Y.-J., Rey, S.-C., Lee, H.-C., \& Walker, A. R. 1999, Multiple Stellar Populations in the Globular Cluster $\omega$ Centauri as Tracers of a Merger Event, Nature, 402, 55
Lee, J.-W., Kang, Y.-W., Lee, J., \& Lee, Y.-W. 2009a, Enrichment by Supernovae in Globular Clusters with Multiple Populations, Nature, 462, 480

Lee, K. H., Lee, H. M., Fahlman, G. G., \& Lee, M. G. 2003, Wide-Field CCD Photometry of the Globular Cluster M92, AJ, 126, 815

Lee, J.-W., Lee, J., Kang, Y.-W., Lee, Y.-W., Han, S.I., Joo, S.-J., Rey, S.-C., \& Yong, D. 2009b, Chemical Inhomogeneity in Red Giant Branch Stars and RR lyrae Variables in NGC 1851: Two Subpopulations in Red Giant Branch, ApJ, 695, L78

Lee, Y.-W., et al. 2005, Super-Helium-Rich Populations and the Origin of Extreme Horizontal-Branch Stars in Globular Clusters, ApJ, 621, L57

Lehmann, I., \& Scholz, R.-D. 1997, Tidal Radii of the Globular Clusters M5, M12, M13, M15, M53, NGC 5053 and NGC 5466 from Automated Star Counts, A\&A, 320, 776

Leon, S., Meylan, G., \& Combes, F. 2000, Tidal Tails around 20 Galactic Globular Clusters. Observational Evidence for Gravitational Disk/Bulge Shocking, A\&A, 359, 907

Lynden-Bell, D. 1982, The Fornax-Leo-Sculptor System, Observatory, 102, 202

Majewski, S. R., Skrutskie, M. F., Weinberg, M. D., \& Ostheimer, J. C. 2003, Two Micron All Sky Survey View of the Sagittarius Dwarf Galaxy. I. Morphology of the Sagittarius Core and Tidal Arms, ApJ, 599, 1082

Marino, A. F., Milone, A. P., Piotto, G., Villanova, S., Bedin, L. R., Bellini, A., \& Renzini, A. 2009, A Double Stellar Generation in the Globular Cluster NGC 6656 (M22). Two Stellar Groups with Different Iron and S-Process Element Abundances, A\&A, 505, 1099

Martin, N. F., Ibata, R. A., Bellazzini, M., Irwin, M. J., Lewis, G. F., \& Dehnen, W. 2004, A Dwarf Galaxy Remnant in Canis Major: the Fossil of an In-Place Accretion on to the Milky Way, MNRAS, 348, 12

McCall, M. L. 2004, On Determining Extinction from Reddening, AJ, 128, 2144

Milone, A. P., et al. 2008, The ACS Survey of Galactic Globular Clusters. III. The Double Subgiant Branch of NGC 1851, ApJ, 673, 241

Minniti, D. 1995, Metal-Rich Globular Clusters with R Less than or Equal $3 \mathrm{kpc}$ : Disk or Bulge Clusters, AJ, 109, 1663

Montuori, M., Capuzzo-Dolcetta, R., Di Matteo, P., Lepinette, A., \& Miocchi, P. 2007, Tidal Tails around Globular Clusters: Are They a Good Tracer of Cluster Orbits?, ApJ, 659, 1212

Moretti, A., et al. 2009, MCAO Near-IR Photometry of the Globular Cluster NGC 6388: MAD Observations in Crowded Fields, A\&A, 493, 539 
Murali, C., \& Weinberg, M. D. 1997a, The Effect of the Galactic Spheroid on Globular Cluster Evolution, MNRAS, 288, 749

Murali, C., \& Weinberg, M. D. 1997b, Evolution of the Galactic Globular Cluster System, MNRAS, 291, 717

Nagashima, C., et al. 1999, Development of SIRUSA Simultaneous-Color infraRed Imager for Unbiased Survey, in Star Formation 1999, ed. T. Nakamoto (Nobeyama: Nobeyama Radio Observatory), 397

Nagayama, T., et al. 2003, SIRUS: a Near Infrared Simultaneous Three-Band Camera, Proc. SPIE, 4841, 459

Nakasato, N., \& Nomoto, K. 2003, Three-Dimensional Simulations of the Chemical and Dynamical Evolution of the Galactic Bulge, ApJ, 588, 842

Newberg, H. J., Willett, B. A., Yanny, B., \& Xu, Y. 2010, The Orbit of the Orphan Stream, ApJ, 711, 32

Newberg, H. J., Yanny, B., \& Willett, B. A. 2009, Discovery of a New, Polar-Orbiting Debris Stream in the Milky Way Stellar Halo, ApJ, 700, L61

Newberg, H. J., et al. 2002, The Ghost of Sagittarius and Lumps in the Halo of the Milky Way, ApJ, 569, 245

Newberg, H. J., et al. 2003, Sagittarius Tidal Debris 90 Kiloparsecs from the Galactic Center, ApJ, 596, L191

Ng, Y. K., \& Bertelli, G. 1996, On the Extinction towards Baade's Window, A\&A, 315, 116

Odenkirchen, M., Grebel, E. K., Kayser, A., Rix, H.W., \& Dehnen, W. 2009, Kinematics of the Tidal Debris of the Globular Cluster Palomar 5, AJ, 137, 3378

Odenkirchen, M., et al. 2001, Detection of Massive Tidal Tails around the Globular Cluster Palomar 5 with Sloan Digital Sky Survey Commissioning Data, ApJ, 548, L165

Odenkirchen, M., et al. 2003, The Extended Tails of Palomar 5: A $10^{\circ}$ Arc of Globular Cluster Tidal Debris, 126, 2385

Olszewski, E. W., Saha, A., Knezek, P., Subramaniam, A., de Boer, T., \& Seitzer, P. 2009, A 500 Parsec Halo Surrounding the Galactic Globular NGC 1851, AJ, 138, 1570

Pancino, E., Ferraro, F. R., Bellazzini, M., Piotto, G., \& Zoccali, M. 2000, New Evidence for the Complex Structure of the Red Giant Branch in $\omega$ Centauri, ApJ, 534, L83

Peterson, C. J., \& Reed, B. C. 1987, Structural Parameters and Luminosities of Globular Clusters, PASP, 99,20

Piotto, G. 2008, Multiple Stellar Populations in Galactic GCs: Observational Evidence, Mem. Soc. Astron. Italiana, 79, 334
Piotto, G., et al. 2007, A Triple Main Sequence in the Globular Cluster NGC 2808, ApJ, 661, L53

Pryor, C., McClure, R. D., Fletcher, J. M., \& Hesser, J. E. 1989, Mass-to-Light Ratios for Globular Clusters.I-The Centrally Concentrated Clusters NGC 6624, M28 (NGC 6626), and M70 (NGC 6681), AJ, 98, 596

Rieke, G. H., \& Lebofsky, M. J. 1985, The Interstellar Extinction Law from 1 to 13 Microns, ApJ, 288, 618

Rocha-Pinto, H. J., Majewski, S. R., Skrutskie, M. F., Crane, J. D., \& Patterson, R. J. 2004, Exploring Halo Substructure with Giant Stars: A Diffuse Star Cloud or Tidal Debris around the Milky Way in Triangulum-Andromeda, ApJ, 615, 732

Rockosi, C. M., et al. 2002, A Matched-Filter Analysis of the Tidal Tails of the Globular Cluster Palomar 5, AJ, 124, 349

Schlegel, D. J., Finkbeiner, D. P., \& Davis, M. 1998, Maps of Dust Infrared Emission for Use in Estimation of Reddening and Cosmic Microwave Background Radiation Foregrounds, ApJ, 500, 525

Siegel, M. H., Majewski, S. R., Cudworth, K. M., \& Takamiya, M. 2001, A Cluster's Last Stand: The Death of Palomar 13, AJ, 121, 935

Skrutskie, M. F., et al. 2006, The Two Micron All Sky Survey (2MASS), AJ, 131, 1163

Sohn, Y.-J., Park, J.-H., Rey, S.-C., Kim, H.-I., Oh, S. J., Lee, S.-G., Lee, M. G., \& Han, W. 2003, Wide-Field Stellar Distributions around the Remote Young Galactic Globular Clusters Palomar 3 and Palomar 4, AJ, 126, 803

Sollima, A., Ferraro, F. R., Pancino, E., \& Bellazzini, M. 2005, On the Discrete Nature of the Red giant Branch of $\omega$ Centauri, MNRAS, 357, 265

Stetson, P. B. 1987, DAOPHOT - A Computer Program for Crowded-Field Stellar Photometry, PASP, 99, 191

Stetson, P. B. 1992, in IAU Colloq. 136, Stellar Photometry: Current Techniques and Future Developments, ed. C. J. Butler \& I. Elliot (Cambridge: Cambridge Univ. Press), 291

Stetson, P. B., \& Harris, W. E. 1988, CCD Photometry of the Globular Cluster M92, AJ, 96, 909

Stetson, P. B., \& West, M. J. 1994, A Color-Magnitude Diagram for NGC 6287: The Oldest Globular Cluster in the Galaxy?, PASP, 106, 726

Testa, V., Zaggia, S. R., Andreon, S., Longo, G., Scaramella, R., Djorgovski, S. G., \& de Carvalho, R. 2000, Use of DPOSS Data to Study Globular Cluster Halos: an Application to M92, A\&A, 356, 127

Trager, S. C., King, I. R., \& Djorgovski, S. 1995, Catalogue of Galactic Globular-Cluster SurfaceBrightness Profiles, AJ, 109, 218 
van den Bergh, S. 1993, The Proto-Galaxy, Globular Clusters, and Quasars, ApJ, 411, 178

Ventura, P., Caloi, V., D'Antona, F., Ferguson, J., Milone, A., \& Piotto, G. P. 2009, The $\mathrm{C}+\mathrm{N}+\mathrm{O}$ Abundances and the Splitting of the Subgiant Branch in the Globular Cluster NGC 1851, MNRAS, 399, 934

Vieira, K., et al. 2007, Proper Motions in the Galactic Bulge: Plaut's Window, AJ, 134, 1432

Villanova, S., et al. 2007, The Multiplicity of the Subgiant Branch of $\omega$ Centauri: Evidence for Prolonged Star Formation, ApJ, 663, 296

Yanny, B., et al. 2000, Identification of A-Colored Stars and Structure in the Halo of the Milky Way from Sloan Digital Sky Survey Commissioning Data, ApJ, 540,825

Yanny, B., et al. 2003, A Low-Latitude-Halo Stream around the Milky Way, ApJ, 588, 824

Yanny, B., et al. 2009, Tracing Sagittarius Structure with SDSS and SEGUE Imaging and Spectroscopy, ApJ, 700, 1282

Yim, K.-J., \& Lee, H. M. 2002, Tidal Tails of Globular Clusters, JKAS, 35, 75

Zoccali, M., et al. 2003, Age and Metallicity Distribution of the Galactic Bulge from Extensive Optical and Near-IR Stellar Photometry, A\&A, 399, 931 